\title{
THE EULER IMPLICIT/EXPLICIT SCHEME FOR THE 2D TIME-DEPENDENT NAVIER-STOKES EQUATIONS WITH SMOOTH OR NON-SMOOTH INITIAL DATA
}

\author{
YINNIAN HE
}

\begin{abstract}
This paper considers the stability and convergence results for the Euler implicit/explicit scheme applied to the spatially discretized twodimensional (2D) time-dependent Navier-Stokes equations. A Galerkin finite element spatial discretization is assumed, and the temporal treatment is implicit/explict scheme, which is implicit for the linear terms and explicit for the nonlinear term. Here the stability condition depends on the smoothness of the initial data $u_{0} \in H^{\alpha}$, i.e., the time step condition is $\tau \leq C_{0}$ in the case of $\alpha=2, \tau|\log h| \leq C_{0}$ in the case of $\alpha=1$ and $\tau h^{-2} \leq C_{0}$ in the case of $\alpha=0$ for mesh size $h$ and some positive constant $C_{0}$. We provide the $H^{2}$-stability of the scheme under the stability condition with $\alpha=0,1,2$ and obtain the optimal $H^{1}-L^{2}$ error estimate of the numerical velocity and the optimal $L^{2}$ error estimate of the numerical pressure under the stability condition with $\alpha=1,2$.
\end{abstract}

\section{INTRODUCTION}

Let $\Omega$ be a bounded domain in $R^{2}$ assumed to have a Lipschitz continuous boundary $\partial \Omega$ and to satisfy a further condition stated in (A1) below. We consider the time-dependent Navier-Stokes problem

$$
\left\{\begin{array}{l}
u_{t}-\nu \Delta u+(u \cdot \nabla) u+\nabla p=f, \operatorname{div} u=0,(x, t) \in \Omega \times(0, T] ; \\
u(x, 0)=u_{0}(x), x \in \Omega ;\left.u(x, t)\right|_{\partial \Omega}=0, t \in[0, T],
\end{array}\right.
$$

where $u=u(x, t)=\left(u_{1}(x, t), u_{2}(x, t)\right)$ represents the velocity vector, $p=p(x, t)$ the pressure, $f=f(x, t)$ the prescribed body force, $u_{0}(x)$ the initial velocity, $\nu>0$ the viscosity, and $T>0$ a finite time.

There are numerous works devoted to the development of efficient schemes for the Navier-Stokes equations [3, 4, 9, 10, 11, 13, 14, 15, 16, 19, 20, 21, 23, 27, 6, 30, 32, 31, 37, fully implicit, semi-implicit and implicit/explicit scheme. A key issue is the stability conditions of schemes. Usually the fully implicit schemes are unconditionally stable. However, at each time step, one has to solve a system of nonlinear equations. An explicit scheme is much easier in computation. But it suffers the severely restricted time step size from stability requirement. A popular approach is based on an implicit scheme for the linear terms and a semi-implicit

Received by the editor February 26, 2007 and, in revised form, September 17, 2007.

2000 Mathematics Subject Classification. Primary 35L70, 65N30, 76D06.

Key words and phrases. Navier-Stokes equations, mixed finite element, Euler implicit/explicit scheme, Smooth or non-smooth initial data.

This research was subsidized by the NSF of China 10671154 and the National Basic Research Program under the grant 2005CB321703. 
scheme or an explicit scheme for the nonlinear term. A semi-implicit scheme for the nonlinear term results in a linear system with a variable coefficient matrix of time, and an explicit treatment for the nonlinear term gives a constant matrix. Stability and convergence conditions of schemes have been studied by many authors. The main results are summarized below, where we set $\Omega \subset R^{d}$ with $d=2$, 3 , and $0<h<1$ denotes the mesh size in the spatial direction and $0<\tau=\frac{T}{N}<1$ denotes the step size in the time direction, which may change. However, $T>0$ is fixed throughout this paper.

- For the Crank-Nicolson scheme (fully implicit), Heywood and Rannacher [23. proved that it is almost unconditionally stable and convergent, i.e. stable and convergent when

$$
\tau \leq C_{0},
$$

for some positive constant $C_{0}$ depending on the data $\left(\nu, \Omega, T, u_{0}, f\right)$ in the case of $d=2,3$.

- For a two-step scheme (semi-implicit), He and Li [14] gave the following convergence condition:

$$
\tau h^{-1 / 2} \leq C_{0}
$$

- For the Crank-Nicolson extrapolation scheme (semi-implicit), He [15] has proved that (1.2) is the stability and convergence condition of the scheme in the case of $d=2$.

- For the Crank-Nicolson/Adams-Bashforth scheme (implicit/explicit), Marion and Temam provided in 32 the following stability condition:

$$
\tau h^{-d} \leq C_{0}, d=2,3,
$$

and recently, Tone [37] proved the convergence under the condition

$$
\tau h^{-2-d / 2} \leq C_{0}, d=2,3 .
$$

- A modified Crank-Nicolson/Adams-Bashforth scheme (implicit/explicit) was proposed by Johnston and Liu [26], in which the nonlinear term and pressure term are discretized explicitly. They claimed in their numerical simulations that the scheme is stable under the standard stability condition

$$
\|u\|_{L^{\infty} \tau h^{-1} \leq 1, d=2,3} .
$$

No theoretical analysis has been given.

- For a three-step backward extrapolating scheme (implicit/explicit), Baker et al. 4] gave the convergence condition

$$
\tau h^{-4 / 7} \leq C_{0},
$$

in the case of $d=2,3$.

- Clearly, the time-step condition

$$
\tau h^{-r} \leq C_{0}
$$

for some $r>0$ was imposed in these previous works when an implicit/ explicit scheme is applied.

Recently, He and Sun [19] have improved the result of (1.8) and proved that the stability and convergence condition of the Crank-Nicolson/Adams-Bashforth scheme is (1.2). 
This paper focuses on the Euler implicit/explicit scheme with a finite element approximation in spatial direction for solving the time-dependent Navier-Stokes equations in the case of $d=2$, which were studied by Marion and Temam [32, Tone [37, Kim and Moin [27] and Issacson and Keller [25. The scheme consists of using a finite element pair $\left(X_{h}, M_{h}\right)$ for the spatial discretization, the implicit scheme for the linear term and the explicit scheme for the nonlinear term for the time discretization. Under the assumptions (A1), (A2) in $\S 2$ with $u^{0} \in D\left(A^{\alpha / 2}\right), \alpha=$ $0,1,2$ and $(\mathrm{A} 3)$ in $\S 3$, we prove that the scheme is stable, i.e.,

$$
\sigma^{2-\alpha}\left(t_{m}\right)\left(\left\|\frac{u_{h}^{m}-u_{h}^{m-1}}{\tau}\right\|_{0}^{2}+\nu^{2}\left\|A_{h} u_{h}^{m}\right\|_{0}^{2}+\left\|p_{h}^{m}\right\|_{0}^{2}\right) \leq \kappa, 1 \leq m \leq N,
$$

when the stability condition

$$
\left\{\begin{array}{l}
\tau \leq C_{0}, \alpha=2 \\
\tau|\log h| \leq C_{0}, \alpha=1 \\
\tau h^{-2} \leq C_{0}, \alpha=0
\end{array}\right.
$$

is satisfied. Under the stability condition (1.10) with $\alpha=1,2$, we also provide the $H^{1}-L^{2}$ optimal error estimate for the numerical velocity and the $L^{2}$-optimal error estimate for the numerical pressure:

$$
\begin{aligned}
\left\|u\left(t_{m}\right)-u_{h}^{m}\right\|_{L^{2}}^{2} & \leq \kappa\left(\sigma^{-(2-\alpha)}\left(t_{m}\right) \tau^{2}+\sigma^{-(2-\alpha)}\left(t_{m}\right) h^{4}\right), \\
\left\|u\left(t_{m}\right)-u_{h}^{m}\right\|_{H^{1}}^{2} & \leq \kappa\left(\sigma^{-(3-\alpha)}\left(t_{m}\right) \tau^{2}+\sigma^{-(2-\alpha)}\left(t_{m}\right) h^{2}\right), \\
\left\|p\left(t_{m}\right)-p_{h}^{m}\right\|_{L^{2}}^{2} & \leq \kappa\left(\sigma^{-(4-\alpha)}\left(t_{m}\right) \tau^{2}+\sigma^{-(3-\alpha)}\left(t_{m}\right) h^{2}\right),
\end{aligned}
$$

for all $1 \leq m \leq N$. Here $\sigma(t)=\min \{1, t\}, \kappa$ is some positive constant depending on the data $\left(\nu, \Omega, T, u_{0}, f\right)$, and $A_{h}$ is a discrete Stokes operator.

Moreover, similar results were proved for the Euler implicit/explicit scheme which is applied to the spatial discretization based on the spectral Galerkin method by $\mathrm{He}[11,12$.

Remark 1.1. In the case of $\alpha=2$, for the first order scheme (the Euler implicit/explicit scheme) we obtain the same $H^{1}$-error bound of the numerical velocity and a better $L^{2}$-error bound of the numerical pressure than the second order scheme (Crank-Nicolson scheme), excepting the $L^{2}$-error estimate for the numerical velocity. Previously, Heywood and Rannacher in [23] provided the following error estimates for the numerical velocity and pressure:

$$
\begin{aligned}
\left\|u\left(t_{m}\right)-u_{h}^{m}\right\|_{H^{1}}^{2} & \leq \kappa\left(\sigma^{-1}\left(t_{m}\right) \tau^{2}+h^{2}\right), 1 \leq m \leq N, \\
\left\|p\left(t_{m}\right)-p_{h}^{m}\right\|_{L^{2}}^{2} & \leq \kappa\left(\sigma^{-3}\left(t_{m}\right) \tau^{2}+\sigma^{-1}\left(t_{m}\right) h^{2}\right), 1 \leq m \leq N,
\end{aligned}
$$

and the $L^{2}$-error estimate for the numerical velocity:

$$
\left\|u\left(t_{m}\right)-u_{h}^{m}\right\|_{L^{2}}^{2} \leq \kappa\left(\sigma^{-2}\left(t_{m}\right) \tau^{4}+h^{4}\right), t_{m} \in(0, T], 1 \leq m \leq N .
$$

This paper is organized as follows. In $\S 2$ an abstract functional setting of the Navier-Stokes problem is given together with some basic assumptions (A1) and (A2) with $\alpha=0,1,2$. In $\S 3$ we set out our assumption (A3) concerning the finite element spaces $X_{h}$ and $M_{h}$, finite element Galerkin approximation in space and some properties on the trilinear form $b(\cdot, \cdot, \cdot)$. Section 3 contains the optimal error estimate and a priori estimate results of the finite element solution $\left(u_{h}(t), p_{h}(t)\right)$. In $\S 4$ we describe the Euler implicit/explicit scheme and prove the stability result of the scheme. In $\S 5$ we describe the dual scheme and prove its stability result. In $\S 6$ we obtain the optimal $H^{1}-L^{2}$-error estimate of the numerical velocity and the 
optimal $L^{2}$-error estimate of the numerical pressure under the stability condition (1.10) with $\alpha=1,2$.

\section{Functional Setting of the Navier-Stokes equations}

For the mathematical setting of problem (1.1), we introduce the following Hilbert spaces:

$$
X=H_{0}^{1}(\Omega)^{2}, Y=L^{2}(\Omega)^{2}, M=L_{0}^{2}(\Omega)=\left\{q \in L^{2}(\Omega) ; \int_{\Omega} q d x=0\right\} .
$$

The space $L^{2}(\Omega)^{d}, d=1,2,4$, is associated with the usual $L^{2}$-scalar product $(\cdot, \cdot)$ and $L^{2}$-norm $\|\cdot\|_{0}$. The space $X$ is associated with its usual scalar product and equivalent norm

$$
((u, v))=(\nabla u, \nabla v),\|u\|_{X}=\|\nabla u\|_{0} .
$$

Next, let the closed subset $V$ of $X$ be given by

$$
V=\{v \in X ; \operatorname{div} v=0\}
$$

and denote by $H$ the closed subset of $Y$, i.e.,

$$
H=\left\{v \in Y ; \operatorname{div} v=0,\left.v \cdot n\right|_{\partial \Omega}=0\right\} .
$$

We refer readers to [1, 10, 22, 36 for details on these spaces. We denote the Stokes operator by $A=-P \Delta$, where $P$ is the $L^{2}$-orthogonal projection of $Y$ onto $H$ and the domain of $A$ by $D(A)=H^{2}(\Omega)^{2} \cap V$. As mentioned above, we need a further assumption on $\Omega$ provided in [23].

(A1) Assume that $\Omega$ is smooth so that the unique solution $(v, q) \in(X, M)$ of the steady Stokes problem

$$
-\nu \Delta v+\nabla q=g, \quad \operatorname{div} v=0 \quad \text { in } \Omega,\left.v\right|_{\partial \Omega}=0,
$$

for any prescribed $g \in Y$, exists and satisfies

$$
\|v\|_{H^{2}}+\|q\|_{H^{1}} \leq c\|g\|_{0}
$$

where $c>0$ is a generic constant depending on $\Omega$ and $\nu$, and may take different values at its different occurrences.

We remark that the validity of assumption (A1) is known (see [10, 22, 28, 36]) if $\partial \Omega$ is of $C^{2}$ or if $\Omega$ is a two-dimensional convex polygon. From the assumption (A1), it is well known [1, 22, 29] that

$$
\begin{aligned}
\|v\|_{H^{2}} & \leq c\|A v\|_{0}, v \in D(A), \\
\|v\|_{0} & \leq \gamma_{0}\|\nabla v\|_{0}, v \in X,\|\nabla v\|_{0} \leq \gamma_{0}\|A v\|_{0}, v \in D(A),
\end{aligned}
$$

where $\gamma_{0}$ is a positive constant depending only on $\Omega$. We usually make the following assumption about the prescribed data for problem (1.1).

(A2) The initial velocity $u_{0}(x)$ and the force $f(x, t)$ are such that $u_{0} \in$ $D\left(A^{\alpha / 2}\right), f, f_{t}, f_{t t} \in L^{\infty}(0, T ; Y)$ with

$$
\left\|A^{\alpha / 2} u_{0}\right\|_{0}+\sup _{0 \leq t \leq T}\left\{\|f(t)\|_{0}+\left\|f_{t}(t)\right\|_{0}+\left\|f_{t t}(t)\right\|_{0}\right\} \leq C
$$

for some positive constant $C$, and $\alpha=0,1,2$, where $D\left(A^{\frac{1}{2}}\right)=V$ and $D\left(A^{0}\right)=H$.

Moreover, we define the continuous bilinear forms $a(\cdot, \cdot)$ and $d(\cdot, \cdot)$ on $X \times X$ and $X \times M$, respectively, by

$$
a(u, v)=\nu((u, v)), u, v \in X, d(v, q)=(q, \operatorname{div} v), v \in X, q \in M,
$$


and a trilinear form on $X \times X \times X$ by

$$
\begin{aligned}
b(u, v, w) & =\left((u \cdot \nabla) v+\frac{1}{2}(\operatorname{div} u) v, w\right) \\
& =\frac{1}{2}((u \cdot \nabla) v, w)-\frac{1}{2}((u \cdot \nabla) w, v), u, v, w \in X .
\end{aligned}
$$

With the above notation, the variational formulation of problem (1.1) reads as follows: Find $(u, p) \in(X, M)$ for all $t \in[0, T]$ such that for all $(v, q) \in(X, M)$,

$$
\begin{aligned}
\left(u_{t}, v\right)+a(u, v)-d(v, p)+d(u, q)+b(u, u, v) & =(f, v), \\
u(0) & =u_{0} .
\end{aligned}
$$

In order to proceed the theoretical and numerical analysis for the variational formulation (2.3)-(2.4), we need to introduce the following existence, uniqueness and modified regularity results.

Theorem 2.1. Under the assumptions (A1) and (A2), the problem (2.3)-(2.4) admits a unique solution $u \in L^{\infty}(0, T ; H) \cap L^{2}(0, T ; V)$ satisfying the following regularities:

$$
\|u(t)\|_{0}^{2}+\sigma^{\frac{1}{2}(1-\alpha)(2-\alpha)}(t)\|\nabla u(t)\|_{0}^{2}+\sigma^{2-\alpha}(t)\left(\|A u(t)\|_{0}^{2}+\|\nabla p(t)\|_{0}^{2}+\left\|u_{t}(t)\right\|_{0}^{2}\right)
$$

$\int_{0}^{t}\left\{\|\nabla u\|_{0}^{2}+\sigma^{\frac{1}{2}(1-\alpha)(2-\alpha)}(s)\left(\left\|u_{t}\right\|_{0}^{2}+\|A u\|_{0}^{2}+\|\nabla p\|_{0}^{2}\right)\right\} d s$

$$
+\int_{0}^{t}\left\{\sigma^{2-\alpha}(s)\left\|\nabla u_{t}\right\|_{0}^{2}+\sigma^{3-\alpha}(s)\left(\left\|u_{t t}\right\|_{0}^{2}+\left\|A u_{t}\right\|_{0}^{2}+\left\|\nabla p_{t}\right\|_{0}^{2}\right)\right\} d s \leq k,
$$

for all $0 \leq t \leq T$.

Proof. For the existence and uniqueness of the solution in the case of $\alpha=0$, the reader may refer to Temam [36]. For the regularity results related to $\alpha=2$, the reader may refer to Heywood and Rannacher [22, and for the regularity results related to $\alpha=1$, the reader may refer to Hill and Süli [24] and He [1] and He et al. [17].

The case $\alpha=0$ has been proved in [12], except for the estimates of $\|\nabla p(t)\|_{0}^{2}$ and $\left\|\nabla p_{t}\right\|_{0}^{2}$. However, these can be done by using (1.1) and some nonlinear term estimates.

\section{Finite element Galerkin approximation}

Let $h>0$ be a real positive parameter. The finite element subspace $\left(X_{h}, M_{h}\right)$ of $(X, M)$ is characterized by $J_{h}=J_{h}(\Omega)$, a partitioning of $\bar{\Omega}$ into triangles $K$ or quadrilaterals $K$, assumed to be uniformly regular as $h \rightarrow 0$. For further details, the reader may refer to Ciarlet [7] and Girault and Raviart [10].

We define the subspace $V_{h}$ of $X_{h}$ given by

$$
V_{h}=\left\{v_{h} \in X_{h} ; d\left(v_{h}, q_{h}\right)=0, \forall q_{h} \in M_{h}\right\}
$$

Let $P_{h}: Y \rightarrow V_{h}$ denote the $L^{2}$-orthogonal projection defined by

$$
\left(P_{h} v, v_{h}\right)=\left(v, v_{h}\right), v \in Y, v_{h} \in V_{h} .
$$


We assume that the couple $\left(X_{h}, M_{h}\right)$ satisfies the following approximation properties:

(A3) For each $v \in H^{2}(\Omega)^{2} \cap X$ and $q \in H^{1}(\Omega) \cap M$, there exist approximations $\pi_{h} v \in X_{h}$ and $\rho_{h} q \in M_{h}$ such that

$$
\left\|\nabla\left(v-\pi_{h} v\right)\right\|_{0} \leq c h\|A v\|_{0},\left\|q-\rho_{h} q\right\|_{0} \leq c h\|\nabla q\|_{0} .
$$

For each $v_{h} \in X_{h}$, one has the inverse inequality

$$
\left\|\nabla v_{h}\right\|_{0} \leq c_{1} h^{-1}\left\|v_{h}\right\|_{0}, v_{h} \in X_{h} ;
$$

and the so-called inf-sup inequality: For each $q_{h} \in M_{h}$, there exists $v_{h} \in X_{h}, v_{h} \neq 0$, such that

$$
d\left(v_{h}, q_{h}\right) \geq c_{2}\left\|q_{h}\right\|_{0}\left\|\nabla v_{h}\right\|_{0},
$$

where $c_{1}$ and $c_{2}$ are positive constants depending on $\Omega$.

We give an example of the spaces $X_{h}$ and $M_{h}$ such that the assumption (A3) is satisfied. Let $\Omega$ be a convex, polygonal domain in plane and $J_{h}=J_{h}(\Omega)$, a partitioning of $\bar{\Omega}$ into triangles $K$, assumed to be uniformly regular as $h \rightarrow 0$. For any nonnegative integer $l$, we denote by $P_{l}(K)$ the space of polynomials of degrees less than or equal to $l$ on $K$.

Example 1 (Girault-Raviart [10]).

$$
\begin{gathered}
X_{h}=\left\{v_{h} \in C^{0}(\Omega)^{2} \cap X ;\left.v_{h}\right|_{K} \in P_{2}(K)^{2}, \forall K \in J_{h}\right\}, \\
M_{h}=\left\{q_{h} \in M ;\left.q_{h}\right|_{K} \in P_{0}(K), \forall K \in J_{h}\right\} .
\end{gathered}
$$

Example 2 (Bercovier-Pironneau [5]). We consider the triangulation $J_{h / 2}$ obtained by dividing each triangle of $J_{h}$ into four triangles (by joining the mid-sides). We set

$$
\begin{gathered}
X_{h}=\left\{v_{h} \in C^{0}(\bar{\Omega})^{2} \cap X ;\left.v_{h}\right|_{K} \in P_{1}(K)^{2}, \quad \forall K \in J_{h / 2}\right\}, \\
M_{h}=\left\{q_{h} \in C^{0}(\bar{\Omega}) \cap M ;\left.q_{h}\right|_{K} \in P_{1}(K), \quad \forall K \in J_{h}\right\} .
\end{gathered}
$$

The following properties are classical (see [2, 10, 22, 24]):

$$
\begin{aligned}
\left\|\nabla P_{h} v\right\|_{0} & \leq c\|\nabla v\|_{0}, v \in X, \\
\left\|v-P_{h} v\right\|_{0}+h\left\|\nabla\left(v-P_{h} v\right)\right\|_{0} & \leq c h^{2}\|A v\|_{0}, v \in D(A), \\
\left\|v-P_{h} v\right\|_{0} & \leq c h\left\|\nabla\left(v-P_{h} v\right)\right\|_{0}, v \in X .
\end{aligned}
$$

The standard finite element Galerkin approximation of (2.3) (2.4) based on $\left(X_{h}, M_{h}\right)$ reads as follows: Find $\left(u_{h}, p_{h}\right) \in\left(X_{h}, M_{h}\right)$ such that for all $0<t \leq T$ and $\left(v_{h}, q_{h}\right) \in\left(X_{h}, M_{h}\right)$,

$$
\begin{aligned}
\left(u_{h t}, v_{h}\right)+a\left(u_{h}, v_{h}\right)-d\left(v_{h}, p_{h}\right)+d\left(u_{h}, q_{h}\right)+b\left(u_{h}, u_{h}, v_{h}\right) & =\left(f, v_{h}\right), \\
u_{h}(0)=u_{0 h} & =P_{h} u_{0} .
\end{aligned}
$$

With the above statements, a discrete analogue $A_{h}=-P_{h} \Delta_{h}$ of the Stokes operator $A$ is defined through the condition that $\left(-\Delta_{h} u_{h}, v_{h}\right)=\left(\left(u_{h}, v_{h}\right)\right)$ for all $u_{h}, v_{h} \in X_{h}$. The restriction of $A_{h}$ to $V_{h}$ is invertible, with the inverse $A_{h}^{-1}$. Since $A_{h}^{-1}$ is self-adjoint and positive definite, we may define "discrete" Sobolev norms on $V_{h}$, of any order $r \in R$, by setting

$$
\left\|v_{h}\right\|_{r}=\left\|A_{h}^{r / 2} v_{h}\right\|_{0}, v_{h} \in V_{h} .
$$


These norms will be assumed to have various properties similar to their continuous counterparts, an assumption that implicitly imposes conditions on the structure of the spaces $X_{h}$ and $M_{h}$. In particular, it holds that

$$
\left\|v_{h}\right\|_{1}=\left\|\nabla v_{h}\right\|_{0},\left\|v_{h}\right\|_{2}=\left\|A_{h} v_{h}\right\|_{0}, v_{h} \in V_{h} .
$$

By the way, we derive from (2.2) that

$$
\left\|v_{h}\right\|_{0} \leq \gamma_{0}\left\|\nabla v_{h}\right\|_{0},\left\|\nabla v_{h}\right\|_{0} \leq \gamma_{0}\left\|A_{h} v_{h}\right\|_{0}, v_{h} \in V_{h}
$$

where $\gamma_{0}>0$ is a constant depending only on $\Omega$.

This section considers preliminary estimates which are useful in the error estimates of finite element solution. Some estimates of the trilinear form $b$ are given in the following lemma and the proof can be found in [15, 16, 24].

Lemma 3.1. The trilinear form $b$ satisfies the following estimates:

$$
\begin{aligned}
b\left(u, v_{h}, w_{h}\right) & =\left((u \cdot \nabla) v_{h}, w_{h}\right)=-\left((u \cdot \nabla) w_{h}, v_{h}\right), \\
b\left(u_{h}, v_{h}, w_{h}\right) & =-b\left(u_{h}, w_{h}, v_{h}\right), \\
\left|b\left(u_{h}, v_{h}, w_{h}\right)\right| & +\left|b\left(v_{h}, u_{h}, w_{h}\right)\right|+\left|b\left(w_{h}, u_{h}, v_{h}\right)\right| \\
& \leq c_{0}|\log h|^{1 / 2}\left\|u_{h}\right\|_{1}\left\|v_{h}\right\|_{1}\left\|w_{h}\right\|_{0}, \\
\left|b\left(u_{h}, v_{h}, w_{h}\right)\right| & +\left|b\left(v_{h}, u_{h}, w_{h}\right)\right|+\left|b\left(w_{h}, u_{h}, v_{h}\right)\right| \\
& \leq \frac{c_{0}}{2}\left\|u_{h}\right\|_{0}^{1 / 2}\left\|u_{h}\right\|_{1}^{1 / 2}\left\|v_{h}\right\|_{1}\left\|w_{h}\right\|_{0}^{1 / 2}\left\|w_{h}\right\|_{1}^{1 / 2} \\
& +\frac{c_{0}}{2}\left\|u_{h}\right\|_{1}\left\|v_{h}\right\|_{0}^{1 / 2}\left\|v_{h}\right\|_{1}^{1 / 2}\left\|w_{h}\right\|_{0}^{1 / 2}\left\|w_{h}\right\|_{1}^{1 / 2},
\end{aligned}
$$

for all $u \in V, u_{h}, v_{h}, w_{h} \in X_{h}$ and

$$
\begin{aligned}
\left|b\left(u_{h}, v_{h}, w_{h}\right)\right| & +\left|b\left(v_{h}, u_{h}, w_{h}\right)\right|+\left|b\left(w_{h}, u_{h}, v_{h}\right)\right| \\
& \leq \frac{1}{2} c_{0}\left\|A_{h} v_{h}\right\|_{0}^{1 / 2}\left\|v_{h}\right\|_{1}^{1 / 2}\left\|u_{h}\right\|_{0}^{1 / 2}\left\|u_{h}\right\|_{1}^{1 / 2}\left\|w_{h}\right\|_{0} \\
& +\frac{1}{2} c_{0}\left\|A_{h} v_{h}\right\|_{0}^{1 / 2}\left\|v_{h}\right\|_{0}^{1 / 2}\left\|u_{h}\right\|_{1}\left\|w_{h}\right\|_{0},
\end{aligned}
$$

for all $u_{h}, v_{h} \in V_{h}, w_{h} \in X_{h}$, where $c_{0}>0$ is a constant depending only on $\Omega$.

Before we proceed further, we need some continuous and discrete GagliardoNirenberg estimates (see Temam [36] and Hill and Süli [24]).

Lemma 3.2. It holds that

$$
\begin{aligned}
\|v\|_{L^{4}} \leq c\|v\|_{0}^{1 / 2}\|\nabla v\|_{0}^{1 / 2}, \forall v \in X,\|\nabla v\|_{L^{4}} & \leq c\|\nabla v\|_{0}^{1 / 2}\|A v\|_{0}^{1 / 2}, \forall v \in D(A), \\
\left\|v_{h}\right\|_{L^{\infty}} \leq c\left\|v_{h}\right\|_{0}^{1 / 2}\left\|A_{h} v_{h}\right\|_{0}^{1 / 2},\left\|v_{h}\right\|_{L^{\infty}} & \leq c|\log h|^{1 / 2}\left\|\nabla v_{h}\right\|_{0}, \forall v_{h} \in V_{h}, \\
\left\|\nabla v_{h}\right\|_{L^{4}} & \leq c\left\|\nabla v_{h}\right\|_{0}^{1 / 2}\left\|A_{h} v_{h}\right\|_{0}^{1 / 2}, \forall v_{h} \in V_{h} .
\end{aligned}
$$

In order to perform our error analysis for time discretization, we recall the following smooth properties of $\left(u_{h}, p_{h}\right)$. 
Theorem 3.3. Assume that assumptions (A1)-(A3) are valid. Then the finite element solution $\left(u_{h}, p_{h}\right)$ satisfies the following estimates:

$$
\begin{array}{r}
\left\|u_{h}(t)\right\|_{0}^{2}+\sigma^{\frac{1}{2}(1-\alpha)(2-\alpha)}(t)\left\|\nabla u_{h}(t)\right\|_{0}^{2}+\sigma^{2-\alpha}(t)\left\|A_{h} u_{h}(t)\right\|_{0}^{2} \\
+\int_{0}^{t}\left\{\left\|\nabla u_{h}\right\|_{0}^{2}+\sigma^{\frac{1}{2}(1-\alpha)(2-\alpha)}(s)\left\|A_{h} u_{h}\right\|_{0}^{2}\right\} d s \leq \kappa, \\
\sigma^{2+r-\alpha}(t)\left\|u_{h t}(t)\right\|_{r}^{2} \leq \kappa, r=0,1,2, \\
\int_{0}^{t}\left\{\sigma^{\frac{1}{2}(1-\alpha)(2-\alpha)}(s)\left\|u_{h t}\right\|_{0}^{2}+\sigma^{1+r-\alpha}(s)\left\|u_{h t}\right\|_{r}^{2}\right\} d s \leq k, r=1,2,
\end{array}
$$

for all $0 \leq t \leq T$.

For the proof of Theorem 3.3 in the case of $\alpha=2$, the reader is referred to Heywood and Rannacher [23] and He and Sun [19]. Theorem 3.3 with $\alpha=1,0$ can be proved in a manner similar to the one used in [23, 19].

Next, we can provide some bounds of the error $\left(u-u_{h}, p-p_{h}\right)$.

Theorem 3.4. Under the assumptions (A1), (A2) with $\alpha=1,2$ and (A3), it holds that

$$
\begin{aligned}
\sigma^{2-\alpha}(t)\left\|u(t)-u_{h}(t)\right\|_{0}^{2} & +h^{2} \sigma^{2-\alpha}(t)\left\|\nabla\left(u(t)-u_{h}(t)\right)\right\|_{0}^{2} \\
& +\sigma^{3-\alpha}(t) h^{2}\left\|p(t)-p_{h}(t)\right\|_{0}^{2} \leq \kappa h^{4}
\end{aligned}
$$

for all $t \in(0, T]$.

Proof. For the case $\alpha=2$, Heywood and Rannacher 22] have proved (3.19). For the case $\alpha=1$, Hill and Süli 24] have proved

$$
\begin{aligned}
\left(\sigma(t)+h^{2}\right)\left\|u(t)-u_{h}(t)\right\|_{0}^{2} & +h^{2} \sigma(t)\left\|\nabla\left(u(t)-u_{h}(t)\right)\right\|_{0}^{2} \\
& +h^{2} \int_{0}^{t}\left\|\nabla\left(u-u_{h}\right)\right\|_{0}^{2} d s \leq \kappa h^{4}
\end{aligned}
$$

for all $t \in(0, T]$.

Hence, it is sufficient to prove

$$
\sigma(t)\left\|p(t)-p_{h}(t)\right\|_{0} \leq \kappa h, \quad \forall t \in(0, T],
$$

for $\alpha=1$.

We set $e_{h}=P_{h} u-u_{h}$ and subtract (3.8) from (2.3) with $v=v_{h}$ to obtain

$$
\begin{aligned}
\left(u_{t}-u_{h t}, v_{h}\right) & +a\left(u-u_{h}, v_{h}\right)-d\left(v_{h}, p-p_{h}\right)+b\left(u, u-u_{h}, v_{h}\right) \\
& +b\left(u-u_{h}, u, v_{h}\right)-b\left(u-u_{h}, u-u_{h}, v_{h}\right)=0, \forall v_{h} \in X_{h} .
\end{aligned}
$$

Taking $v_{h}=2 e_{h t} \in V_{h}$ in (3.22) yields

$$
\begin{aligned}
2\left\|e_{h t}\right\|_{0}^{2} & +2 \nu \frac{d}{d t}\left\|\nabla\left(u-u_{h}\right)\right\|_{0}^{2}+2 b\left(u, u-u_{h}, e_{h t}\right) \\
& +2 b\left(u-u_{h}, u, e_{h t}\right)-2 b\left(u-u_{h}, u-u_{h}, e_{h t}\right) \\
& =2 a\left(u-u_{h}, u_{t}-P_{h} u_{t}\right)+2 \frac{d}{d t} d\left(e_{h}, p-\rho_{h} p\right)-2 d\left(e_{h}, p_{t}-\rho_{h} p_{t}\right) .
\end{aligned}
$$


Due to (2.2), (3.2)-(3.3), (3.6) and Lemmas 3.1 and 3.2, we have

$$
\begin{aligned}
2 a\left(u-u_{h}, u_{t}-P_{h} u_{t}\right) & \leq 2 \nu\left\|\nabla\left(u-u_{h}\right)\right\|_{0}\left\|\nabla\left(u_{t}-P_{h} u_{t}\right)\right\|_{0} \\
& \leq c h\left\|\nabla\left(u-u_{h}\right)\right\|_{0}\left\|A u_{t}\right\|_{0}, \\
2\left|d\left(e_{h}, p_{t}-\rho_{h} p_{t}\right)\right| & \leq 2 \sqrt{2}\left\|\nabla e_{h}\right\|_{0}\left\|p_{t}-\rho_{h} p_{t}\right\|_{0} \\
& \leq c h\left(\left\|\nabla\left(u-u_{h}\right)\right\|_{0}+h\|A u\|_{0}\right)\left\|\nabla p_{t}\right\|_{0}, \\
2\left|b\left(u, u-u_{h}, e_{h t}\right)\right| & +2\left|b\left(u-u_{h}, u, e_{h t}\right)\right| \\
& \leq 4\left(\|u\|_{L^{\infty}}\left\|\nabla\left(u-u_{h}\right)\right\|_{0}+\|\nabla u\|_{L^{4}}\left\|u-u_{h}\right\|_{L^{4}}\right)\left\|e_{h t}\right\|_{0} \\
& \leq \frac{1}{2}\left\|e_{h t}\right\|_{0}^{2}+c\|A u\|_{0}^{2}\left\|\nabla\left(u-u_{h}\right)\right\|_{0}^{2}, \\
2\left|b\left(u-u_{h}, u-u_{h}, e_{h t}\right)\right| & \leq c\left\|\nabla\left(u-u_{h}\right)\right\|_{0}^{2}\left\|\nabla e_{h t}\right\|_{0} \\
& \leq \frac{1}{2}\left\|e_{h t}\right\|_{0}^{2}+c h^{-2}\left\|\nabla\left(u-u_{h}\right)\right\|_{0}^{4} .
\end{aligned}
$$

Combining this inequality with (3.23) gives

$$
\begin{aligned}
\left\|e_{h t}\right\|_{0}^{2} & +2 \nu \frac{d}{d t}\left\|\nabla\left(u-u_{h}\right)\right\|_{0}^{2} \leq 2 \frac{d}{d t} d\left(e_{h}, p-\rho_{h} p\right) \\
& +c h\left\|\nabla\left(u-u_{h}\right)\right\|_{0}\left\|A u_{t}\right\|_{0}+c h\left(\left\|\nabla\left(u-u_{h}\right)\right\|_{0}+h\|A u\|_{0}\right)\left\|\nabla p_{t}\right\|_{0} \\
& +c\left(\|A u\|_{0}^{2}+h^{-2}\left\|\nabla\left(u-u_{h}\right)\right\|_{0}^{2}\right)\left\|\nabla\left(u-u_{h}\right)\right\|_{0}^{2} .
\end{aligned}
$$

Multiplying (3.24) by $\sigma(t)$, and integrating with respect to time and then using Theorem 2.1 and (3.20), we obtain

$$
\begin{aligned}
\int_{0}^{t} \sigma(s)\left\|e_{h t}\right\|_{0}^{2} d s & \leq 2 \sigma(t) d\left(e_{h}(t), p(t)-\rho_{h} p(t)\right) \\
& +2 \int_{0}^{t}\left|d\left(e_{h}, p-\rho_{h} p\right)\right| d s+2 \nu \int_{0}^{t}\left\|\nabla\left(u-u_{h}\right)\right\|_{0}^{2} d s+\kappa h^{2} \\
& \leq c \sigma(t) h\left(\left\|\nabla\left(u-u_{h}\right)\right\|_{0}+h\|A u\|_{0}\right)\|\nabla p\|_{0} \\
& +h \int_{0}^{t}\left(\left\|\nabla\left(u-u_{h}\right)\right\|_{0}+h\|A u\|_{0}\right)\|\nabla p\|_{0} d s+\kappa h^{2} \leq \kappa h^{2}
\end{aligned}
$$

for all $t \in(0, T]$.

Differentiating (3.22) with respect to time gives

$$
\begin{aligned}
\left(u_{t t}-u_{h t t}, v_{h}\right) & +a\left(u_{t}-u_{h t}, v_{h}\right)-d\left(v_{h}, p_{t}-\rho_{h} p_{t}\right)+b\left(u_{t}, u-u_{h}, v_{h}\right) \\
& +b\left(u-u_{h}, u_{t}, v_{h}\right)+b\left(u, u_{t}-u_{h t}, v_{h}\right)+b\left(u_{t}-u_{h t}, u, v_{h}\right) \\
(3.26) & -b\left(u_{t}-u_{h t}, u-u_{h}, v_{h}\right)-b\left(u-u_{h}, u_{t}-u_{h t}, v_{h}\right)=0, \forall v_{h} \in V_{h} .
\end{aligned}
$$

Taking $v_{h}=2 e_{h t} \in V_{h}$ in (3.26) and using Lemma 3.1, one finds

$$
\begin{aligned}
\frac{d}{d t}\left\|e_{h t}\right\|_{0}^{2} & +\nu\left\|\nabla\left(u_{t}-u_{h t}\right)\right\|_{0}^{2}+\nu\left\|\nabla e_{h t}\right\|_{0}^{2}+2 b\left(u_{t}, u-u_{h}, e_{h t}\right)+2 b\left(u-u_{h}, u_{t}, e_{h t}\right) \\
& +2 b\left(u, u_{t}-P_{h} u_{t}, e_{h t}\right)+2 b\left(u_{t}-P_{h} u_{t}, u, e_{h t}\right)+2 b\left(e_{h t}, u_{h}, e_{h t}\right) \\
& -2 b\left(u_{t}-P_{h} u_{t}, u-u_{h}, e_{h t}\right)-2 b\left(u-u_{h}, u_{t}-P_{h} u_{t}, e_{h t}\right) \\
(3.27) \quad & =\nu\left\|\nabla\left(u_{t}-P_{h} u_{t}\right)\right\|_{0}^{2}+2 d\left(e_{h t}, p_{t}-\rho_{h} p_{t}\right) .
\end{aligned}
$$


Due to (2.2), (3.2), (3.6) and Lemma 3.1, we have

$$
\begin{aligned}
2\left|b\left(u_{t}, u-u_{h}, e_{h t}\right)\right| & +2\left|b\left(u-u_{h}, u_{t}, e_{h t}\right)\right| \\
& \leq 8 \gamma_{0}\left\|\nabla u_{t}\right\|_{0}\left\|\nabla\left(u-u_{h}\right)\right\|_{0}\left\|\nabla e_{h t}\right\|_{0} \\
& \leq \frac{\nu}{8}\left\|\nabla e_{h t}\right\|_{0}^{2}+c\left\|\nabla u_{t}\right\|_{0}^{2}\left\|\nabla\left(u-u_{h}\right)\right\|_{0}^{2}, \\
2\left|b\left(e_{h t}, u_{h}, e_{h t}\right)\right| & \leq 4\left\|e_{h t}\right\|_{0}^{1 / 2}\left\|\nabla e_{h t}\right\|_{0}^{3 / 2}\left\|u_{h}\right\|_{0}^{1 / 2}\left\|\nabla u_{h}\right\|_{0}^{1 / 2} \\
& \leq \frac{\nu}{8}\left\|\nabla e_{h t}\right\|_{0}^{2}+c\left\|u_{h}\right\|_{0}^{2}\left\|\nabla u_{h}\right\|_{0}^{2}\left\|e_{h t}\right\|_{0}^{2}, \\
2\left|b\left(u, u_{t}-P_{h} u_{t}, e_{h t}\right)\right| & +2\left|b\left(u_{t}-P_{h} u_{t}, u, e_{h t}\right)\right| \\
& \leq 8 \gamma_{0}\left\|\nabla\left(u_{t}-P_{h} u_{t}\right)\right\|_{0}\|\nabla u\|_{0}\left\|\nabla e_{h t}\right\|_{0} \\
& \leq \frac{\nu}{8}\left\|\nabla e_{h t}\right\|_{0}^{2}+c h^{2}\|\nabla u\|_{0}^{2}\left\|A u_{t}\right\|_{0}^{2}, \\
2\left|b\left(u_{t}-P_{h} u_{t}, u-u_{h}, e_{h t}\right)\right| & +2\left|b\left(u-u_{h}, u_{t}-P_{h} u_{t}, e_{h t}\right)\right| \\
& \leq 8 \gamma_{0}\left\|\nabla\left(u-u_{h}\right)\right\|\left\|\nabla\left(u_{t}-P_{h} u_{t}\right)\right\|_{0}\left\|\nabla e_{h t}\right\|_{0} \\
& \leq \frac{\nu}{8}\left\|\nabla e_{h t}\right\|_{0}^{2}+c h^{2}\left\|\nabla\left(u-u_{h}\right)\right\|_{0}^{2}\left\|A u_{t}\right\|_{0}^{2}, \\
\nu\left\|\nabla\left(u_{t}-P_{h} u_{t}\right)\right\|_{0}^{2} & +2 d\left(e_{h t}, p_{t}-\rho_{h} p_{t}\right) \leq c h^{2}\left\|A u_{t}\right\|_{0}^{2}+c h\left\|\nabla e_{h t}\right\|_{0}\left\|\nabla p_{t}\right\|_{0} \\
& \leq \frac{\nu}{8}\left\|\nabla e_{h t}\right\|_{0}^{2}+c h^{2}\left(\left\|A u_{t}\right\|_{0}^{2}+\left\|\nabla p_{t}\right\|_{0}^{2}\right) .
\end{aligned}
$$

Combining (3.27) with the above estimates yields

$$
\begin{aligned}
\frac{d}{d t}\left\|e_{h t}\right\|_{0}^{2} & \leq c\left\|u_{h}\right\|_{0}^{2}\left\|\nabla u_{h}\right\|_{0}^{2}\left\|e_{h t}\right\|_{0}^{2}+c\left\|\nabla u_{t}\right\|_{0}^{2}\left\|\nabla\left(u-u_{h}\right)\right\|_{0}^{2} \\
& +c h^{2}\left(1+\|\nabla u\|_{0}^{2}+\left\|\nabla u_{h}\right\|_{0}^{2}\right)\left(\left\|A u_{t}\right\|_{0}^{2}+\left\|\nabla p_{t}\right\|_{0}^{2}\right), .
\end{aligned}
$$

Multiplying (3.28) by $\sigma^{2}(t)$, and integrating with respect to time, we obtain

$$
\begin{aligned}
\sigma^{2}(t)\left\|e_{h t}(t)\right\|_{0}^{2} & \leq c \int_{0}^{t} \sigma(s)\left(1+\left\|u_{h}\right\|_{0}^{2}\left\|\nabla u_{h}\right\|_{0}^{2}\right)\left\|e_{h t}\right\|_{0}^{2} d s \\
& +c \int_{0}^{t} \sigma^{2}(s)\left\|\nabla u_{t}\right\|_{0}^{2}\left\|\nabla\left(u-u_{h}\right)\right\|_{0}^{2} d s \\
& +c h^{2} \int_{0}^{t} \sigma^{2}(s)\left(1+\left\|\nabla u_{h}\right\|_{0}^{2}+\|\nabla u\|_{0}^{2}\right)\left(\left\|A u_{t}\right\|_{0}^{2}+\left\|\nabla p_{t}\right\|_{0}^{2}\right) d s .
\end{aligned}
$$

Using (3.20), (3.25), Theorem 2.1 and Theorem 3.3 in (3.29), we obtain

$$
\sigma^{2}(t)\left\|e_{h t}(t)\right\|_{0}^{2} \leq \kappa h^{2},
$$

which yields

$$
\begin{aligned}
\sigma^{2}(t)\left\|u_{t}-u_{h t}\right\|_{0}^{2} & \leq 2 \sigma^{2}(t)\left\|e_{h t}(t)\right\|_{0}^{2}+2 \sigma^{2}(t)\left\|u_{t}(t)-P_{h} u_{t}(t)\right\|_{0}^{2} \\
& \leq 2 \sigma^{2}(t)\left\|e_{h t}(t)\right\|_{0}^{2}+c h^{2} \sigma^{2}(t)\left\|\nabla u_{t}(t)\right\|_{0}^{2} \leq \kappa h^{2} .
\end{aligned}
$$

Finally, by using (2.2), (3.2), (3.4), (3.22) and Lemma 3.2, one finds

$$
\begin{aligned}
\sigma(t)\left\|p(t)-p_{h}(t)\right\|_{0} & \leq \sigma(t)\left(\left\|\rho_{h} p(t)-p(t)\right\|_{0}+\left\|\rho_{h} p(t)-p_{h}(t)\right\|_{0}\right) \\
& \leq c \sigma(t)\left\|u_{t}(t)-u_{h t}(t)\right\|_{0}+c h \sigma(t)\|\nabla p(t)\|_{0} \\
& +c \sigma(t)\left(1+\|\nabla u\|_{0}+\left\|\nabla\left(u-u_{h}\right)\right\|_{0}\right)\left\|\nabla\left(u(t)-u_{h}(t)\right)\right\|_{0} .
\end{aligned}
$$

Using (3.20), (3.30) and Theorem 2.1 in (3.31), we obtain (3.21). 
We will frequently use a discrete version of the Gronwall lemmas used in 13 and [34].

Lemma 3.5. Let $C$, $\tau$, and $a_{n}, b_{n}, d_{n}$, for integers $n \geq 0$, be nonnegative numbers such that

$$
a_{m}+\tau \sum_{n=1}^{m} b_{n} \leq \tau \sum_{n=0}^{m-1} a_{n} d_{n}+C, m \geq 1
$$

Then

$$
a_{m}+\tau \sum_{n=1}^{m} b_{n} \leq C \exp \left(\tau \sum_{n=0}^{m-1} d_{n}\right), m \geq 1 .
$$

Theorem 3.6. Under the assumptions (A1), (A2) with $\alpha=1,2$ and (A3), $u_{\text {htt }}$ and $u_{\text {httt }}$ satisfy the following bounds:

$$
\begin{aligned}
& \int_{0}^{t} \sigma^{3-r-\alpha}(s)\left\|A_{h}^{-r / 2} u_{h t t}\right\|_{0}^{2} d s \leq \kappa, r=0,1,2, \alpha=1 \text { or } r=0,1, \alpha=2, \\
& \sigma^{4-\alpha}(t)\left\|u_{h t t}(t)\right\|_{0}^{2}+\int_{0}^{t} \sigma^{4-\alpha}(s)\left(\left\|u_{h t t}\right\|_{1}^{2}+\left\|A_{h}^{-1 / 2} u_{h t t t}\right\|_{0}^{2}\right) d s \leq \kappa,
\end{aligned}
$$

for all $0 \leq t \leq T$.

Proof. Differentiating (3.8) with respect to $t$ gives

$$
\left(u_{h t t}, v_{h}\right)+a\left(u_{h t}, v_{h}\right)+b\left(u_{h t}, u_{h}, v_{h}\right)+b\left(u_{h}, u_{h t}, v_{h}\right)=\left(f_{t}, v_{h}\right),
$$

for all $v_{h} \in V_{h}$.

In view of (3.10) and Lemma 3.1, we deduce from (3.36) that

$$
\left\|A_{h}^{-r / 2} u_{h t t}\right\|_{0} \leq\left(\nu+c_{0} \gamma_{0}\left\|\nabla u_{h}\right\|_{0}\right)\left\|A_{h}^{1-r / 2} u_{h t}\right\|_{0}+\gamma_{0}^{r}\left\|f_{t}\right\|_{0},
$$

which yields

$$
\begin{aligned}
\int_{0}^{t} \sigma^{3-r-\alpha}(s)\left\|A_{h}^{-r / 2} u_{h t t}\right\|_{0}^{2} d s & \leq c \int_{0}^{t}\left(1+\left\|\nabla u_{h}\right\|_{0}^{2}\right) \sigma^{3-r-\alpha}(s)\left\|A_{h}^{1-r / 2} u_{h t}\right\|_{0}^{2} d s \\
& +c \int_{0}^{t}\left\|f_{t}\right\|_{0}^{2} d s,
\end{aligned}
$$

for $r=0,1,2, \alpha=1$ or $r=0,1, \alpha=2$. Using Theorem 3.3 in (3.37) gives (3.34).

Furthermore, by differentiating (3.36) with respect to $t$ gives

$$
\begin{aligned}
\left(u_{h t t t}, v_{h}\right) & +a\left(u_{h t t}, v_{h}\right)+2 b\left(u_{h t}, u_{h t}, v_{h}\right)+b\left(u_{h t t}, u_{h}, v_{h}\right) \\
& +b\left(u_{h}, u_{h t t}, v_{h}\right)=\left(f_{t t}, v_{h}\right),
\end{aligned}
$$

for all $v_{h} \in V_{h}$.

Taking $v_{h}=2 u_{h t t}$ in (3.38) and using (3.10) and Lemma 3.1, we deduce

$$
\begin{aligned}
\frac{d}{d t}\left\|u_{h t t}\right\|_{0}^{2} & +2 \nu\left\|u_{h t t}\right\|_{1}^{2}+4 b\left(u_{h t}, u_{h t}, u_{h t t}\right)+2 b\left(u_{h t t}, u_{h}, u_{h t t}\right) \\
& \leq \frac{\nu}{4}\left\|u_{h t t}\right\|_{1}^{2}+4 \nu^{-1} \gamma_{0}^{2}\left\|f_{t t}\right\|_{0}^{2} .
\end{aligned}
$$


In view of (3.10) and Lemma 3.1, we deduce from (3.36) that

$$
\begin{aligned}
4\left|b\left(u_{h t}, u_{h t}, u_{h t t}\right)\right| & \leq 2 c_{0} \gamma_{0}\left\|u_{h t}\right\|_{1}^{2}\left\|u_{h t t}\right\|_{1} \\
& \leq \frac{\nu}{4}\left\|u_{h t t}\right\|_{1}^{2}+4 \nu^{-1} c_{0}^{2} \gamma_{0}^{2}\left\|u_{h t}\right\|_{1}^{4}, \\
2\left|b\left(u_{h t t}, u_{h}, u_{h t t}\right)\right| & \leq c_{0} \gamma_{0}\left\|u_{h t t}\right\|_{0}\left\|u_{h t t}\right\|_{1}\left\|A_{h} u_{h}\right\|_{0} \\
& \leq \frac{\nu}{4}\left\|u_{h t t}\right\|_{1}^{2}+\nu^{-1} c_{0}^{2} \gamma_{0}^{2}\left\|A_{h} u_{h}\right\|_{0}^{2}\left\|u_{h t t}\right\|_{0}^{2} .
\end{aligned}
$$

Combining these inequalities with (3.39) gives

$$
\begin{aligned}
\frac{d}{d t}\left\|u_{h t t}\right\|_{0}^{2} & +\nu\left\|u_{h t t}\right\|_{1}^{2} \leq 4 \nu^{-1} \gamma_{0}^{2}\left\|f_{t t}\right\|_{0}^{2} \\
& +4 \nu^{-1} c_{0}^{2} \gamma_{0}^{2}\left\|u_{h t}\right\|_{1}^{4}+\nu^{-1} c_{0}^{2} \gamma_{0}^{2}\left\|A_{h} u_{h}\right\|_{0}^{2}\left\|u_{h t t}\right\|_{0}^{2} .
\end{aligned}
$$

Multiplying (3.40) by $\sigma^{4-\alpha}(t)$ yields

$$
\begin{aligned}
& \frac{d}{d t}\left(\sigma^{4-\alpha}(t)\left\|u_{h t t}\right\|_{0}^{2}\right)+\nu \sigma^{4-\alpha}(t)\left\|u_{h t t}\right\|_{1}^{2} \leq 4 \nu^{-1} \gamma_{0}^{2}\left\|f_{t t}\right\|_{0}^{2} \\
& \quad+c \sigma^{4-\alpha}(t)\left\|u_{h t}\right\|_{1}^{4}+\left(4-\alpha+c \sigma(t)\left\|A_{h} u_{h}\right\|_{0}^{2}\right) \sigma^{3-\alpha}(t)\left\|u_{h t t}\right\|_{0}^{2} .
\end{aligned}
$$

Integrating (3.41) from 0 to $t$ and using (3.34) and Theorem 3.3, we deduce

$$
\sigma^{4-\alpha}(t)\left\|u_{h t t}\right\|_{0}^{2}+\nu \int_{0}^{t} \sigma^{4-\alpha}(s)\left\|u_{h t t}\right\|_{1}^{2} d s \leq \kappa, \forall t \in(0, T] .
$$

Finally, it follows from (3.38), (3.10) and Lemma 3.1 that

$$
\begin{aligned}
\int_{0}^{t} \sigma^{4-\alpha}(s)\left\|A_{h}^{-1 / 2} u_{h t t t}\right\|_{0}^{2} d s & \leq c \int_{0}^{t}\left(1+\left\|u_{h}\right\|_{1}^{2}\right) \sigma^{4-\alpha}(s)\left\|u_{h t t}\right\|_{1}^{2} d s \\
& +c \int_{0}^{t}\left\{\sigma^{4-\alpha}(s)\left\|u_{h t}\right\|_{1}^{4}+\left\|f_{t t}\right\|_{0}^{2}\right\} d s .
\end{aligned}
$$

Using Theorem 3.3 in (3.43), together with (3.42), gives (3.35) for $\alpha=1,2$.

\section{The Euler implicit/eXplicit scheme}

In this section we consider the time discretization of the finite element Galerkin approximation (3.8)-(3.9). Usually for the fully implicit scheme, at each time step, one has to solve a system of nonlinear equations. An explicit scheme is much easier in computation. But it suffers the severely restricted time step size from stability requirement. A popular approach is based on an implicit scheme for the linear terms and an explicit scheme for the nonlinear term. An explicit scheme for the nonlinear term results in a linear system with a constant coefficient matrix such that the computation is easy and the time step restriction is $\tau \leq C_{0}$ which will be proved in this section and Section 6 .

Let $t_{n}=n \tau(n=0,1, \ldots, N), \tau=\frac{T}{N}$ the time step size, and $N$ an integer. We define $u_{h}^{0}=u_{0 h}=P_{h} u_{0}$ and $\left(u_{h}^{n}, p_{h}^{n}\right) \in\left(X_{h}, M_{h}\right)$ by the Euler implicit/explicit scheme:

(4.1) $\left(d_{t} u_{h}^{n}, v_{h}\right)+a\left(u_{h}^{n}, v_{h}\right)-d\left(v_{h}, p_{h}^{n}\right)+d\left(u_{h}^{n}, q_{h}\right)+b\left(u_{h}^{n-1}, u_{h}^{n-1}, v_{h}\right)=\left(f\left(t_{n}\right), v_{h}\right)$, here $d_{t} u_{h}^{n}=\frac{1}{\tau}\left(u_{h}^{n}-u_{h}^{n-1}\right)$.

We see from (3.3) and (3.5)-(3.6) that

$$
\left\|u_{h}^{0}\right\|_{\alpha}=\left\|u_{0 h}\right\|_{\alpha}=\left\|P_{h} u_{0}\right\|_{\alpha} \leq c_{\alpha}\left\|A^{\alpha / 2} u_{0}\right\|_{0},
$$

if $u_{0} \in D\left(A^{\alpha / 2}\right)$ for some constants $c_{\alpha}$ with $\alpha=0,1,2$. 
The following theorem provides the stability of the scheme (4.1).

Theorem 4.1. Suppose that the assumptions (A1)-(A3) are valid and $0<\tau<1$ satisfies the following stability condition:

$$
G_{h} \tau \leq \nu, G_{h}=\left\{\begin{array}{l}
4^{2} \nu^{-3 / 2} c_{0}^{2} \gamma_{0} \kappa_{1}^{1 / 2} \kappa_{2}^{1 / 2}, \alpha=2 \\
4^{2} c_{0}^{2} \nu^{-1} \kappa_{1}|\log h|, \alpha=1 \\
4^{2} c_{0}^{2} c_{1}^{2} \kappa_{0} h^{-2}, \alpha=0
\end{array}\right.
$$

Then the following hold:

$$
\begin{gathered}
\left\|u_{h}^{m}\right\|_{0}^{2}+\nu \tau \sum_{n=1}^{m}\left\|u_{h}^{n}\right\|_{1}^{2} \leq \kappa_{0}, \\
\tau \sum_{n=1}^{m} \sigma^{\frac{1}{2}(1-\alpha)(2-\alpha)}\left(t_{n}\right)\left(\nu^{2}\left\|A_{h} u_{h}^{n}\right\|_{0}^{2}+\nu\left\|d_{t} u_{h}^{n}\right\|_{1}^{2} \tau+\left\|d_{t} u_{h}^{n}\right\|_{0}^{2}\right) \\
+\sigma^{\frac{1}{2}(1-\alpha)(2-\alpha)}\left(t_{m}\right) \nu\left\|u_{h}^{m}\right\|_{1}^{2} \leq \kappa_{1}, \\
\sigma^{2-\alpha}\left(t_{m}\right)\left(\left\|d_{t} u_{h}^{m}\right\|_{0}^{2}+\left\|p_{h}^{m}\right\|_{0}^{2}\right)+\nu \tau \sum_{n=1}^{m} \sigma^{2-\alpha}\left(t_{n}\right)\left\|d_{t} u_{h}^{n}\right\|_{1}^{2} \leq \kappa_{2}, \\
\sigma^{2-\alpha}\left(t_{m}\right) \nu^{2}\left\|A_{h} u_{h}^{m}\right\|_{0}^{2} \leq \kappa_{2},
\end{gathered}
$$

for all $0 \leq m \leq N$, where $\kappa_{\alpha} \geq c_{\alpha}^{2} \nu^{\alpha}\left\|A^{\alpha / 2} u_{0}\right\|_{0}^{2}$ are some positive constants depending on the data $\left(\nu, \Omega, T, u_{0}, f\right)$.

Proof. First, taking $v_{h}=2 u_{h}^{n} \tau \in V_{h}$ and $v_{h}=A_{h} u_{h}^{n} \tau+\nu^{-1} d_{t} u_{h}^{n} \tau \in V_{h}$, respectively, and $q_{h}=0$ in (4.1) and using (3.12) and the relation

$$
2(x-y) x=|x|^{2}-|y|^{2}+|x-y|^{2}, \forall x, y \in R^{2}
$$

we obtain

$$
\begin{aligned}
\left\|u_{h}^{n}\right\|_{0}^{2} & -\left\|u_{h}^{n-1}\right\|_{0}^{2}+\left\|d_{t} u_{h}^{n}\right\|_{0}^{2} \tau^{2}+2 \nu\left\|u_{h}^{n}\right\|_{1}^{2} \tau+2 b\left(u_{h}^{n-1}, u_{h}^{n}, d_{t} u_{h}^{n}\right) \tau^{2} \\
& =2\left(f\left(t_{n}\right), u_{h}^{n}\right) \tau \\
\left\|u_{h}^{n}\right\|_{1}^{2} & -\left\|u_{h}^{n-1}\right\|_{1}^{2}+\left\|d_{t} u_{h}^{n}\right\|_{1}^{2} \tau^{2}+\nu^{-1}\left\|d_{t} u_{h}^{n}\right\|_{0}^{2} \tau+\nu\left\|A_{h} u_{h}^{n}\right\|_{0}^{2} \tau \\
& +b\left(u_{h}^{n-1}, u_{h}^{n-1}, A_{h}^{r} u_{h}^{n}+\nu^{-1} d_{t} u_{h}^{n}\right) \tau \\
& =\left(f\left(t_{n}\right), A_{h} u_{h}^{n}+\nu^{-1} d_{t} u_{h}^{n}\right) \tau .
\end{aligned}
$$

In view of Lemma 3.1 and (3.10), it holds that

$$
\begin{aligned}
2\left|b\left(u_{h}^{n-1}, u_{h}^{n-1}, u_{h}^{n}\right)\right| \tau & =2\left|b\left(u_{h}^{n-1}, u_{h}^{n}, d_{t} u_{h}^{n}\right)\right| \tau^{2} \leq \frac{1}{2} G^{1 / 2}\left(u_{h}^{n-1}\right)\left\|u_{h}^{n}\right\|_{1}\left\|d_{t} u_{h}^{n}\right\|_{0} \tau^{2} \\
& \leq \frac{1}{2}\left\|d_{t} u_{h}^{n}\right\|_{0}^{2} \tau^{2}+\frac{1}{4} G\left(u_{h}^{n-1}\right)\left\|u_{h}^{n}\right\|_{1}^{2} \tau^{2}, \\
2\left|\left(f\left(t_{n}\right), u_{h}^{n}\right)\right| \tau & \leq \frac{\nu}{4}\left\|u_{h}^{n}\right\|_{1}^{2} \tau+4 \nu^{-1} \gamma_{0}^{2}\left\|f\left(t_{n}\right)\right\|_{0}^{2} \tau,
\end{aligned}
$$


and

$$
\begin{aligned}
& \left|b\left(u_{h}^{n-1}, u^{n}-u_{h}^{n-1}, A_{h} u_{h}^{n}+\nu^{-1} d_{t} u_{h}^{n}\right)\right| \tau \\
& \quad \leq \frac{1}{2} G^{1 / 2}\left(u_{h}^{n-1}\right)\left\|d_{t} u_{h}^{n}\right\|_{1}\left\|A_{h} u_{h}^{n}+\nu^{-1} d_{t} u_{h}^{n}\right\|_{0} \tau^{2} \\
& \quad \leq \frac{1}{2}\left\|d_{t} u_{h}^{n}\right\|_{1}^{2} \tau^{2}+\frac{1}{4} G\left(u_{h}^{n-1}\right)\left(\left\|A_{h} u_{h}^{n}\right\|_{0}^{2}+\nu^{-2}\left\|d_{t} u_{h}^{n}\right\|_{0}^{2}\right) \tau^{2}, \\
& \quad\left|b\left(u_{h}^{n-1}, u_{h}^{n}, A_{h} u_{h}^{n}+\nu^{-1} d_{t} u_{h}^{n}\right)\right| \tau \\
& \quad \leq c_{0}\left(\left\|u_{h}^{n-1}\right\|_{0}^{1 / 2}\left\|u_{h}^{n-1}\right\|_{1}^{1 / 2}\left\|u_{h}^{n}\right\|_{1}^{1 / 2}+\left\|u_{h}^{n-1}\right\|_{1}\left\|u_{h}^{n}\right\|_{0}^{1 / 2}\right) \\
& \quad \times\left\|A_{h} u_{h}^{n}\right\|_{0}^{1 / 2}\left(\left\|A_{h} u_{h}^{n}\right\|_{0}+\nu^{-1}\left\|d_{t} u_{h}^{n}\right\|_{0}\right) \tau \\
& \quad \leq \frac{\nu}{8}\left\|A_{h} u_{h}^{n}\right\|_{0}^{2} \tau+\frac{1}{8 \nu}\left\|d_{t} u_{h}^{n}\right\|_{0}^{2} \tau \\
& \quad+2\left(\frac{4}{\nu}\right)^{3} c_{0}^{4}\left(\left\|u_{h}^{n-1}\right\|_{0}^{2}\left\|u_{h}^{n}\right\|_{1}^{2}+\left\|u_{h}^{n-1}\right\|_{1}^{2}\left\|u_{h}^{n}\right\|_{0}^{2}\right)\left\|u_{h}^{n-1}\right\|_{1}^{2} \tau, \\
& \left|\left(f\left(t_{n}\right), A_{h} u_{h}^{n}+\nu^{-1} d_{t} u_{h}^{n}\right)\right| \tau \leq \frac{\nu}{8}\left\|A_{h} u_{h}^{n}\right\|_{0}^{2} \tau+\frac{1}{8 \nu}\left\|d_{t} u_{h}^{n}\right\|_{0}^{2} \tau+4 \nu^{-1}\left\|f\left(t_{n}\right)\right\|_{0}^{2} \tau,
\end{aligned}
$$

where

$$
G\left(u_{h}^{n}\right)=\left\{\begin{array}{l}
4^{2} c_{0}^{2} \gamma_{0}\left\|u_{h}^{n}\right\|_{1}\left\|A_{h} u_{h}^{n}\right\|_{0}, \quad \alpha=2, \\
4^{2} c_{0}^{2}|\log h|\left\|u_{h}^{n}\right\|_{1}^{2}, \quad \alpha=1, \\
4^{2} c_{0}^{2} c_{1}^{2} h^{-2}\left\|u_{h}^{n}\right\|_{0}^{2}, \quad \alpha=0 .
\end{array}\right.
$$

Combining these inequalities with (4.8) and (4.9) yields

$$
\begin{aligned}
\left\|u_{h}^{n}\right\|_{0}^{2} & -\left\|u_{h}^{n-1}\right\|_{0}^{2}+\frac{1}{2}\left\|d_{t} u_{h}^{n}\right\|_{0}^{2} \tau^{2}+\nu\left\|u_{h}^{n}\right\|_{1}^{2} \tau+\frac{1}{2}\left(\nu-G\left(u_{h}^{n-1}\right) \tau\right)\left\|u_{h}^{n}\right\|_{1}^{2} \tau \\
& \leq 4 \nu^{-1} \gamma_{0}^{2}\left\|f\left(t_{n}\right)\right\|_{0}^{2} \tau, \\
2 \nu\left\|u_{h}^{n}\right\|_{1}^{2} & -2 \nu\left\|u_{h}^{n-1}\right\|_{1}^{2}+\left\|d_{t} u_{h}^{n}\right\|_{1}^{2} \tau^{2}+\left\|d_{t} u_{h}^{n}\right\|_{0}^{2} \tau+\nu^{2}\left\|A_{h} u_{h}^{n}\right\|_{0}^{2} \tau \\
& +\frac{\nu}{2}\left(\nu-G\left(u_{h}^{n-1}\right) \tau\right)\left(\left\|A_{h} u_{h}^{n}\right\|_{0}^{2}+\nu^{-2}\left\|d_{t} u_{h}^{n}\right\|_{0}^{2}\right) \tau \\
& \leq d_{n-1} \nu\left\|u_{h}^{n-1}\right\|_{1}^{2} \tau+8 \nu^{-1}\left\|f\left(t_{n}\right)\right\|_{0}^{2} \tau,
\end{aligned}
$$

where

$$
d_{n-1}=4\left(\frac{4}{\nu}\right)^{3} c_{0}^{4}\left(\left\|u_{h}^{n-1}\right\|_{0}^{2}\left\|u_{h}^{n}\right\|_{1}^{2}+\left\|u_{h}^{n-1}\right\|_{1}^{2}\left\|u_{h}^{n}\right\|_{0}^{2}\right) .
$$

Now, we define $d_{t} u_{h}^{0}=\lim _{t \rightarrow 0} u_{h t}(t)$ through (3.8), i.e.,

$$
\left(d_{t} u_{h}^{0}, v_{h}\right)+a\left(u_{h}^{0}, v_{h}\right)+b\left(u_{h}^{0}, u_{h}^{0}, v_{h}\right)=\left(f\left(t_{0}\right), v_{h}\right),
$$

for all $v_{h} \in V_{h}$. Then, we deduce from (4.1) and (4.13) that

$$
\left(d_{t t} u_{h}^{1}, v_{h}\right)+a\left(d_{t} u_{h}^{1}, v_{h}\right)=\frac{1}{\tau} \int_{t_{0}}^{t_{1}}\left(f_{t}(t), v_{h}\right) d t,
$$

and

$$
\begin{aligned}
\left(d_{t t} u_{h}^{n}, v_{h}\right) & +a\left(d_{t} u_{h}^{n}, v_{h}\right)+b\left(d_{t} u_{h}^{n-1}, u_{h}^{n-1}, v_{h}\right)+b\left(u_{h}^{n-2}, d_{t} u_{h}^{n-1}, v_{h}\right) \\
& =\frac{1}{\tau} \int_{t_{n-1}}^{t_{n}}\left(f_{t}(t), v_{h}\right) d t
\end{aligned}
$$


for all $2 \leq n \leq N$. Hence, it follows from (4.14) that

$$
\left\|d_{t} u_{h}^{1}\right\|_{0}^{2}+\left\|d_{t t} u_{h}^{1}\right\|_{0}^{2} \tau^{2}+\nu\left\|d_{t} u_{h}^{1}\right\|_{1}^{2} \tau \leq\left\|d_{t} u_{h}^{0}\right\|_{0}^{2}+\frac{\gamma_{0}^{2}}{\nu} \int_{t_{0}}^{t_{1}}\left\|f_{t}\right\|_{0}^{2} d t .
$$

Next, by taking $v_{h}=2 d_{t} u_{h}^{n} \tau$ in (4.15) and using (3.12), we deduce

$$
\begin{aligned}
\left\|d_{t} u_{h}^{n}\right\|_{0}^{2} & -\left\|d_{t} u_{h}^{n-1}\right\|_{0}^{2}+\left\|d_{t t} u_{h}^{n}\right\|_{0}^{2} \tau^{2}+2 \nu\left\|d_{t} u_{h}^{n}\right\|_{1}^{2} \tau \\
& +2 b\left(d_{t} u_{h}^{n-1}, u_{h}^{n-1}, d_{t} u_{h}^{n}\right) \tau+2 b\left(u_{h}^{n-2}, d_{t} u_{h}^{n}, d_{t t} u_{h}^{n}\right) \tau^{2} \\
& =2\left(\int_{t_{n-1}}^{t_{n}} f_{t}(t), d_{t} u_{h}^{n}\right) d t .
\end{aligned}
$$

In view of Lemma 3.1 and (3.10), it holds that

$$
\begin{aligned}
2\left|b\left(d_{t} u_{h}^{n}, u_{h}^{n-1}, d_{t} u_{h}^{n}\right)\right| \tau & \leq c_{0}\left\|u_{h}^{n-1}\right\|_{0}^{1 / 2}\left\|u_{h}^{n-1}\right\|_{1}^{1 / 2}\left\|d_{t} u_{h}^{n}\right\|_{0}^{1 / 2}\left\|d_{t} u_{h}^{n}\right\|_{1}^{3 / 2} \tau \\
& \leq \frac{\nu}{4}\left\|d_{t} u_{h}^{n}\right\|_{1}^{2} \tau+\left(\frac{2}{\nu}\right)^{3} c_{0}^{4}\left\|u_{h}^{n-1}\right\|_{0}^{2}\left\|u_{h}^{n-1}\right\|_{1}^{2}\left\|d_{t} u_{h}^{n}\right\|_{0}^{2} \tau, \\
2\left|b\left(d_{t} u^{n}-d_{t} u_{h}^{n-1}, u_{h}^{n-1}, d_{t} u_{h}^{n}\right)\right| \tau & \leq \frac{1}{2} G^{1 / 2}\left(u_{h}^{n-1}\right)\left\|d_{t} u_{h}^{n}\right\|_{1}\left\|d_{t t} u_{h}^{n}\right\|_{0} \tau^{2} \\
& \leq \frac{1}{4}\left\|d_{t t} u_{h}^{n}\right\|_{0}^{2} \tau^{2}+\frac{1}{4} G\left(u_{h}^{n-1}\right)\left\|d_{t} u_{h}^{n}\right\|_{1}^{2} \tau^{2}, \\
2\left|b\left(u_{h}^{n-2}, d_{t} u^{n}, d_{t t} u_{h}^{n}\right)\right| \tau^{2} & \leq \frac{1}{2} G^{1 / 2}\left(u_{h}^{n-2}\right)\left\|d_{t} u_{h}^{n}\right\|_{1}\left\|d_{t t} u_{h}^{n}\right\|_{0} \tau^{2} \\
& \leq \frac{1}{4}\left\|d_{t t} u_{h}^{n}\right\|_{0}^{2} \tau^{2}+\frac{1}{4} G\left(u_{h}^{n-2}\right)\left\|d_{t} u_{h}^{n}\right\|_{1}^{2} \tau^{2}, \\
2\left|\int_{t_{n-1}}^{t_{n}}\left(f_{t}(t), d_{t} u_{h}^{n}\right) d t\right| & \leq \frac{\nu}{4}\left\|d_{t} u_{h}^{n}\right\|_{1}^{2} \tau+\frac{4 \gamma_{0}^{2}}{\nu} \int_{t_{n-1}}^{t_{n}}\left\|f_{t}(t)\right\|_{0}^{2} d t .
\end{aligned}
$$

Combining these inequalities with (4.17) yields

$$
\begin{aligned}
\left\|d_{t} u_{h}^{n}\right\|_{0}^{2} & -\left\|d_{t} u_{h}^{n-1}\right\|_{0}^{2}+\frac{1}{2}\left\|d_{t t} u_{h}^{n}\right\|_{0}^{2} \tau^{2}+\nu\left\|d_{t} u_{h}^{n}\right\|_{1}^{2} \tau \\
& +\frac{1}{4}\left(2 \nu-G\left(u_{h}^{n-1}\right) \tau-G\left(u_{h}^{n-2}\right) \tau\right)\left\|d_{t} u_{h}^{n}\right\|_{1}^{2} \tau \\
& \leq 2\left(\frac{4}{\nu}\right)^{3} c_{0}^{4}\left\|u_{h}^{n-1}\right\|_{0}^{2}\left\|u_{h}^{n-1}\right\|_{1}^{2}\left\|d_{t} u_{h}^{n}\right\|_{0}^{2} \tau+\frac{4 \gamma_{0}^{2}}{\nu} \int_{t_{n-1}}^{t_{n}}\left\|f_{t}(t)\right\|_{0}^{2} d t
\end{aligned}
$$

for all $2 \leq n \leq N$.

Next, we deduce from (4.1) and Lemma 3.1 that

$2 \nu\left\|A_{h} u_{h}^{n}\right\|_{0} \leq 2\left\|d_{t} u^{n}\right\|_{0}+2\left\|f\left(t_{n}\right)\right\|_{0}+c_{0}\left\|u_{h}^{n-1}\right\|_{0}^{1 / 2}\left\|u_{h}^{n-1}\right\|_{1}\left\|A_{h} u_{h}^{n-1}\right\|_{0}^{1 / 2}$

$$
\leq 2\left\|d_{t} u^{n}\right\|_{0}+2\left\|f\left(t_{n}\right)\right\|_{0}+\nu\left\|A_{h} u_{h}^{n-1}\right\|_{0}+\nu^{-1} c_{0}^{2}\left\|u_{h}^{n-1}\right\|_{0}\left\|u_{h}^{n-1}\right\|_{1}^{2} .
$$

Moreover, we deduce from (2.2), (3.4), (4.1) and Lemma 3.1 that

$$
\left\|p_{h}^{n}\right\|_{0} \leq c \nu\left\|u_{h}^{n}\right\|_{1}+c\left\|d_{t} u^{n}\right\|_{0}+c\left\|f\left(t_{n}\right)\right\|_{0}+c\left\|u_{h}^{n-1}\right\|_{1}^{2} .
$$

Now, we will prove (4.4)-(4.6) by induction. For $\alpha=0,1,2$, we deduce from (4.3) that

$$
G\left(u_{h}^{0}\right) \tau \leq G_{h} \tau \leq \nu
$$

Due to (4.2), (4.4)-(4.6) hold for $m=0$. For $\alpha=0,1$, we can obtain (4.4)-(4.6) with $m=1$ by using (4.11)-(4.12), (4.20)-(4.21). For $\alpha=2$, (4.13) and Lemma 3.1 
can yield

$$
\left\|d_{t} u_{h}^{0}\right\|_{0} \leq 2 \nu\left\|A_{h} u_{h}^{0}\right\|_{0}+\left\|f\left(t_{0}\right)\right\|_{0}+G^{1 / 2}\left(u_{h}^{0}\right)\left\|u_{h}^{0}\right\|_{1} .
$$

Hence, we imply (4.4)-(4.6) with $m=1$ by using (4.2), (4.11)-(4.12), (4.16) and (4.19)-(4.22). Assuming that (4.4)-(4.6) hold for $m=0,1, \ldots, J$, we want to prove that they hold for $m=J+1$.

Proof of (4.4). In view of the induction assumption and (4.3), it holds that

$$
G\left(u_{h}^{n-1}\right) \tau \leq G_{h} \tau \leq \nu, 1 \leq n \leq J+1, G\left(u_{h}^{n-2}\right) \tau \leq G_{h} \tau \leq \nu, 2 \leq n \leq J,
$$

for $\alpha=0,1,2$. Summing (4.11) from $n=1$ to $J+1$ and using (4.23), we obtain (4.4) for $m=J+1$ in the case of $\alpha=0,1,2$.

Proof of (4.5). For $\alpha=1,2$, by summing (4.12) from $n=1$ to $n=J+1$ and using (4.23), we obtain

$$
\begin{aligned}
\nu\left\|u_{h}^{J+1}\right\|_{1}^{2} & +\tau \sum_{n=1}^{J+1}\left(\left\|d_{t} u_{h}^{n}\right\|_{0}^{2}+\nu\left\|d_{t} u_{h}^{n}\right\|_{1}^{2} \tau+\nu^{2}\left\|A_{h} u_{h}^{n}\right\|_{0}^{2}\right) \\
& \leq \tau \sum_{n=0}^{J} d_{n} \nu\left\|u_{h}^{n}\right\|_{1}^{2}+8 \nu^{-1} T \sup _{0 \leq t \leq T}\|f(t)\|_{0}^{2}+2 \nu\left\|u_{h}^{0}\right\|_{1}^{2} .
\end{aligned}
$$

We set

$$
\begin{aligned}
& a_{n}=\nu\left\|u_{h}^{n}\right\|_{1}^{2}, C=8 \nu^{-1} T \sup _{0 \leq t \leq T}\|f(t)\|_{0}^{2}+2 \nu\left\|u_{h}^{0}\right\|_{1}^{2}, \\
& b_{n}=\left\|d_{t} u_{h}^{n}\right\|_{0}^{2}+\nu\left\|d_{t} u_{h}^{n}\right\|_{1}^{2} \tau+\nu^{2}\left\|A_{h} u_{h}^{n}\right\|_{0}^{2} .
\end{aligned}
$$

Applying Lemma 3.5 to (4.24) and using (4.4), we obtain (4.5) with $m=J+1$.

For $\alpha=0$, multiplying (4.12) by $\sigma\left(t_{n}\right)$, using (4.23) and noting $\sigma\left(t_{n}\right) \leq \sigma\left(t_{n-1}\right)+$ $\tau$, which will often be used later, we obtain

$$
\begin{gathered}
2 \sigma\left(t_{n}\right) \nu\left\|u_{h}^{n}\right\|_{1}^{2}-2 \nu \sigma\left(t_{n-1}\right)\left\|u_{h}^{n-1}\right\|_{1}^{2}+\sigma\left(t_{n}\right)\left(\left\|d_{t} u_{h}^{n}\right\|_{0}^{2}+\nu\left\|d_{t} u_{h}^{n}\right\|_{1}^{2} \tau+\nu^{2}\left\|A_{h} u_{h}\right\|_{0}^{2}\right) \tau \\
\quad \leq 2 \nu\left\|u_{h}^{n-1}\right\|_{1}^{2} \tau+d_{n-1}\left(\tau+\sigma\left(t_{n-1}\right)\right) \nu\left\|u_{h}^{n-1}\right\|_{1}^{2} \tau+8 \nu^{-1}\left\|f\left(t_{n}\right)\right\|_{0}^{2} \tau,
\end{gathered}
$$

for all $1 \leq n \leq J+1$. Summing (4.25) from $n=1$ to $n=J+1$, we deduce

$$
\begin{aligned}
\sigma\left(t_{J+1}\right) \nu\left\|u_{h}^{J+1}\right\|_{1}^{2} & +\tau \sum_{n=1}^{J+1} \sigma\left(t_{n}\right)\left(\left\|d_{t} u_{h}^{n}\right\|_{0}^{2}+\nu\left\|d_{t} u_{h}^{n}\right\|_{1}^{2} \tau+\nu^{2}\left\|A_{h} u_{h}^{n}\right\|_{0}^{2}\right) \\
& \leq 4 \tau \sum_{n=0}^{J} d_{n} \sigma\left(t_{n}\right) \nu\left\|u_{h}^{n}\right\|_{1}^{2}+2 \nu \tau \sum_{n=0}^{J}\left\|u_{h}^{n}\right\|_{1}^{2} \\
& +8 \nu^{-1} T \sup _{0 \leq t \leq T}\|f(t)\|_{0}^{2}+2 \nu \tau^{2} d_{0}\left\|u_{h}^{1}\right\|_{1}^{2}
\end{aligned}
$$

Setting

$$
\begin{gathered}
a_{n}=\sigma\left(t_{n}\right) \nu\left\|u_{h}^{n}\right\|_{1}^{2}, C=8 \nu^{-1} T \sup _{0 \leq t \leq T}\|f(t)\|_{0}^{2} \tau, \\
b_{n}=\sigma\left(t_{n}\right)\left(\left\|d_{t} u_{h}^{n}\right\|_{0}^{2}+\nu\left\|d_{t} u_{h}^{n}\right\|_{1}^{2} \tau+\nu^{2}\left\|A_{h} u_{h}^{n}\right\|_{0}^{2}\right) .
\end{gathered}
$$

Applying Lemma 3.5 to (4.26) and using (4.3)-(4.4), we arrive at (4.5) for $m=J+1$. 
Proof of (4.6). If $\left\|A_{h} u_{h}^{J+1}\right\|_{0} \leq\left\|A_{h} u_{h}^{J}\right\|_{0}$, then the induction assumption yields

$$
\sigma^{2-\alpha}(t) \nu^{2}\left\|A_{h} u_{h}^{J+1}(t)\right\|_{0}^{2} \leq \kappa_{2}, 1 \leq J \leq N-1,
$$

for $\alpha=0,1,2$. Hence, we always assume that

$$
\left\|A_{h} u_{h}^{J+1}\right\|_{0} \geq\left\|A_{h} u_{h}^{J}\right\|_{0}, 1 \leq J \leq N-1 .
$$

For $\alpha=2$, summing (4.18) from $n=2$ to $n=J+1$, adding (4.16) and using (4.4)-(4.5) and (4.23), we deduce

$$
\begin{aligned}
\left\|d_{t} u_{h}^{J+1}\right\|_{0}^{2} & +\tau \sum_{n=1}^{J+1}\left(\nu\left\|d_{t} u_{h}^{n}\right\|_{1}^{2}+\left\|d_{t t} u_{h}^{n}\right\|_{0}^{2}\right) \\
& \leq 2\left(\frac{4}{\nu}\right)^{4} c_{0}^{4} \kappa_{0} \kappa_{1}^{2}+\frac{8 \gamma_{0}^{2}}{\nu} \int_{0}^{T}\left\|f_{t}(t)\right\|_{0}^{2} d t+2\left\|d_{t} u_{h}^{0}\right\|_{0}^{2} .
\end{aligned}
$$

Thus, by combining (4.27)-(4.28) with (4.19)-(4.20) with $n=J+1$, we deduce (4.6) for $m=J+1$.

For $\alpha=1$, by multiplying (4.18) by $\sigma\left(t_{n}\right)$ and summing from $n=2$ to $n=J+1$ and using (4.12) with $n=1$, we find

$$
\begin{aligned}
\sigma\left(t_{J+1}\right)\left\|d_{t} u_{h}^{J+1}\right\|_{0}^{2} & +\nu \tau \sum_{n=1}^{J+1} \sigma\left(t_{n}\right)\left\|d_{t} u_{h}^{n}\right\|_{1}^{2} \\
& \leq \tau \sum_{n=1}^{J+1}\left(1+2\left(\frac{4}{\nu}\right)^{3} c_{0}^{4}\left\|u_{h}^{n-1}\right\|_{0}^{2}\left\|u_{h}^{n-1}\right\|_{1}^{2}\right)\left\|d_{t} u_{h}^{n}\right\|_{0}^{2} \\
& +\frac{4 \gamma_{0}^{2}}{\nu} \int_{0}^{T}\left\|f_{t}(t)\right\|_{0}^{2} d t+2\left(1+d_{0} \tau\right) \nu\left\|u_{h}^{0}\right\|_{1}^{2}+16 \nu^{-1}\left\|f\left(t_{1}\right)\right\|_{0}^{2} \tau .
\end{aligned}
$$

Now, by using (4.27), (4.24) and (4.19)-(4.20) in (4.29), we obtain (4.6) for $m=$ $J+1$.

Finally, for $\alpha=0$, by multiplying (4.18) by $\sigma^{2}\left(t_{n}\right)$, noting $\sigma^{2}\left(t_{n}\right) \leq \sigma^{2}\left(t_{n-1}\right)+$ $3 \sigma\left(t_{n-1}\right) \tau$, which will often be used later, summing from $n=2$ to $n=J+1$ and using (4.12) with $n=1$, we find

$$
\begin{aligned}
\sigma^{2}\left(t_{J+1}\right)\left\|d_{t} u_{h}^{J+1}\right\|_{0}^{2} & +\nu \tau \sum_{n=1}^{J+1} \sigma^{2}\left(t_{n}\right)\left\|d_{t} u_{h}^{n}\right\|_{1}^{2} \\
& \leq \tau \sum_{n=1}^{J+1} \sigma\left(t_{n}\right)\left(2+2\left(\frac{4}{\nu}\right)^{3} c_{0}^{4}\left\|u_{h}^{n-1}\right\|_{0}^{2}\left\|u_{h}^{n-1}\right\|_{1}^{2}\right)\left\|d_{t} u_{h}^{n}\right\|_{0}^{2} \\
& +\frac{4 \gamma_{0}^{2}}{\nu} T \sup _{0 \leq t \leq T}\left\|f_{t}(t)\right\|_{0}^{2}+2\left(1+d_{0} \tau\right) \nu\left\|u_{h}^{0}\right\|_{1}^{2}+16 \nu^{-1}\left\|f\left(t_{1}\right)\right\|_{0}^{2} .
\end{aligned}
$$

Hence, by using (4.30), (4.26)-(4.27) and (4.19)-(4.20), we obtain (4.6) for $m=$ $J+1$.

Theorem 4.2. Under the assumptions of Theorem 4.1, it holds that

$$
\begin{aligned}
\sigma^{3-\alpha}\left(t_{m}\right)\left\|d_{t} u_{h}^{m}\right\|_{1}^{2} & +\nu \tau \sum_{n=2}^{m} \sigma^{3-\alpha}\left(t_{n}\right)\left\|A_{h} d_{t} u_{h}^{n}\right\|_{0}^{2} \leq \kappa_{3}, \\
\tau \sum_{n=2}^{m} \sigma^{3-\alpha}\left(t_{n}\right)\left\|d_{t t} u_{h}^{n}\right\|_{0}^{2} & \leq \kappa_{4}
\end{aligned}
$$


for all $2 \leq m \leq N$ and $\alpha=1,2$, where $\kappa_{3}$ and $\kappa_{4}$ are some positive constants depending on the data $\left(\nu, \Omega, T, u_{0}, f\right)$.

Proof. First, taking $v_{h}=2 A_{h} d_{t} u_{h}^{n} \tau \in V_{h}$ in (4.15), we deduce

$$
\begin{aligned}
\left\|d_{t} u_{h}^{n}\right\|_{1}^{2} & -\left\|d_{t} u_{h}^{n-1}\right\|_{1}^{2}+\left\|d_{t t} u_{h}^{n}\right\|_{1}^{2} \tau^{2}+2 \nu\left\|A_{h} d_{t} u_{h}^{n}\right\|_{0}^{2} \tau \\
& +2 b\left(d_{t} u_{h}^{n-1}, u_{h}^{n-1}, A_{h} d_{t} u_{h}^{n}\right) \tau+2 b\left(u_{h}^{n-2}, d_{t} u_{h}^{n-1}, A_{h} d_{t} u_{h}^{n}\right) \tau \\
& =2 \int_{t_{n-1}}^{t_{n}}\left(f_{t}(t), A_{h} d_{t} u_{h}^{n}\right) d t
\end{aligned}
$$

In view of Lemma 3.1 and (3.10), it holds that

$$
\begin{aligned}
& 2\left|b\left(d_{t} u_{h}^{n-1}, u_{h}^{n-1}, A_{h} d_{t} u_{h}^{n}\right)\right| \tau \leq 2 c_{0} \gamma_{0}\left\|A_{h} u_{h}^{n-1}\right\|_{0}\left\|d_{t} u_{h}^{n-1}\right\|_{1}\left\|A_{h} d_{t} u_{h}^{n}\right\|_{0} \tau \\
& \quad \leq \frac{\nu}{8}\left\|A_{h} d_{t} u_{h}^{n}\right\|_{0}^{2} \tau+8 \nu^{-1} c_{0}^{2} \gamma_{0}^{2}\left\|A_{h} u_{h}^{n-1}\right\|_{0}^{2}\left\|d_{t} u_{h}^{n-1}\right\|_{1}^{2} \tau, \\
& 2\left|b\left(u_{h}^{n-2}, d_{t} u_{h}^{n}, A_{h} d_{t} u_{h}^{n}\right)\right| \tau \leq 2 c_{0} \gamma_{0}^{1 / 2}\left\|u_{h}^{n-2}\right\|_{1}\left\|d_{t} u_{h}^{n}\right\|_{1}^{1 / 2}\left\|A_{h} d_{t} u_{h}^{n}\right\|_{0}^{3 / 2} \tau \\
& \leq \frac{\nu}{2}\left\|A_{h} d_{t} u_{h}^{n}\right\|_{0}^{2} \tau+2\left(\frac{2}{\nu}\right)^{3} c_{0}^{4} \gamma_{0}^{2}\left\|u_{h}^{n-2}\right\|_{1}^{4}\left\|d_{t} u_{h}^{n}\right\|_{1}^{2} \tau, \\
& 2\left|b\left(u_{h}^{n-2}, d_{t} u_{h}^{n}-d_{t} u_{h}^{n-1}, A_{h} d_{t} u_{h}^{n}\right)\right| \tau \leq \frac{1}{2} G^{1 / 2}\left(u_{h}^{n-2}\right)\left\|d_{t t} u_{h}^{n}\right\|_{1}\left\|A_{h} d_{t} u_{h}^{n}\right\|_{0} \tau^{2} \\
& \leq \frac{1}{2}\left\|d_{t t} u_{h}^{n}\right\|_{1}^{2} \tau^{2}+\frac{1}{4} G\left(u_{h}^{n-2}\right)\left\|A_{h} d_{t} u_{h}^{n}\right\|_{0}^{2} \tau^{2}, \\
& 2\left|\int_{t_{n-1}}^{t_{n}}\left(f_{t}(t), A_{h} d_{t} u_{h}^{n}\right) d t\right| \leq \frac{\nu}{8}\left\|A_{h} d_{t} u_{h}^{n}\right\|_{0}^{2} \tau+\frac{8}{\nu} \int_{t_{n-1}}^{t_{n}}\left\|f_{t}(t)\right\|_{0}^{2} d t .
\end{aligned}
$$

Combining these inequalities with (4.33) yields

$$
\begin{aligned}
\left\|d_{t} u_{h}^{n}\right\|_{1}^{2} & -\left\|d_{t} u_{h}^{n-1}\right\|_{1}^{2}+\nu\left\|A_{h} d_{t} u_{h}^{n}\right\|_{0}^{2} \tau+\frac{1}{4}\left(\nu-G\left(u_{h}^{n-2}\right) \tau\right)\left\|A_{h} d_{t} u_{h}^{n}\right\|_{0}^{2} \tau \\
& \leq 8 \nu^{-1} c_{0}^{2} \gamma_{0}^{2}\left\|A_{h} u_{h}^{n-1}\right\|_{0}^{2}\left\|d_{t} u_{h}^{n-1}\right\|_{1}^{2} \tau+2\left(\frac{2}{\nu}\right)^{3} c_{0}^{4} \gamma_{0}^{2}\left\|u_{h}^{n-2}\right\|_{1}^{4}\left\|d_{t} u_{h}^{n}\right\|_{1}^{2} \tau \\
& +\frac{8}{\nu} \int_{t_{n-1}}^{t_{n}}\left\|f_{t}(t)\right\|_{0}^{2} d t,
\end{aligned}
$$

for all $2 \leq n \leq N$. Multiplying (4.34) by $\sigma^{3-\alpha}\left(t_{n}\right)$ and using (4.23), we deduce

$$
\begin{aligned}
\sigma^{3-\alpha}\left(t_{n}\right)\left\|d_{t} u_{h}^{n}\right\|_{1}^{2} & -\sigma^{3-\alpha}\left(t_{n-1}\right)\left\|d_{t} u_{h}^{n-1}\right\|_{1}^{2}+\nu \sigma^{3-\alpha}\left(t_{n}\right)\left\|A_{h} d_{t} u_{h}^{n}\right\|_{0}^{2} \tau \\
& \leq c \sigma^{3-\alpha}\left(t_{n-1}\right)\left\|A_{h} u_{h}^{n-1}\right\|_{0}^{2}\left\|d_{t} u_{h}^{n-1}\right\|_{1}^{2} \tau \\
& +c \sigma^{3-\alpha}\left(t_{n}\right)\left\|u_{h}^{n-2}\right\|_{1}^{4}\left\|d_{t} u_{h}^{n}\right\|_{0}^{2} \tau \\
& +c \sigma^{2-\alpha}\left(t_{n-1}\right)\left\|d_{t} u_{h}^{n-1}\right\|_{1}^{2} \tau+c \int_{t_{n-1}}^{t_{n}}\left\|f_{t}(t)\right\|_{0}^{2} d t,
\end{aligned}
$$

for all $2 \leq n \leq N$. Summing (4.35) from $n=2$ to $n=m$ and using (4.6), we obtain (4.31).

Then, we deduce from (4.15), (3.10) and Lemma 3.1 that

$$
\begin{aligned}
\left\|d_{t t} u_{h}^{n}\right\|_{0} & \leq \nu\left\|A_{h} d_{t} u_{h}^{n}\right\|_{0}+c\left\|d_{t} u_{h}^{n-1}\right\|_{1}\left(\left\|A_{h} u_{h}^{n-1}\right\|_{0}+H(n-3)\left\|A_{h} u_{h}^{n-2}\right\|_{0}\right) \\
& +\frac{1}{2} H(2-n) G^{1 / 2}\left(u_{h}^{0}\right)\left\|d_{t} u_{h}^{1}\right\|_{1}+\tau^{-1 / 2}\left(\int_{t_{n-1}}^{t_{n}}\left\|f_{t}\right\|_{0}^{2} d t\right)^{1 / 2}
\end{aligned}
$$


for all $2 \leq n \leq N$, where $H(t)=1$, as $t \geq 0$ and $H(t)=0$, as $t<0$. Thus, we deduce from (4.36) that

$$
\begin{aligned}
& \sigma^{3-\alpha}\left(t_{n}\right)\left\|d_{t t} u_{h}^{n}\right\|_{0}^{2} \tau \\
& \quad \leq c \sigma^{3-\alpha}\left(t_{n}\right)\left\|A_{h} d_{t} u_{h}^{n}\right\|_{0}^{2} \tau+H(2-n) G\left(u_{h}^{0}\right)(2 \tau)^{3-\alpha}\left\|d_{t} u_{h}^{1}\right\|_{1}^{2} \tau+c \int_{t_{n-1}}^{t_{n}}\left\|f_{t}\right\|_{0}^{2} d t \\
& \quad+c \sigma^{2-\alpha}\left(t_{n-1}\right)\left\|d_{t} u_{h}^{n-1}\right\|_{1}^{2}\left(\sigma\left(t_{n-1}\right)\left\|A_{h} u_{h}^{n-1}\right\|_{0}^{2}+H(n-3) \sigma\left(t_{n-2}\right)\left\|A_{h} u_{h}^{n-2}\right\|_{0}^{2}\right) \tau .
\end{aligned}
$$

Summing the above inequality from $n=2$ to $n=m$ and using Theorem 4.1, (4.21) and (4.31), we get (4.32).

\section{Dual Euler scheme: Stability analysis}

In order to derive the $L^{2}$-bound on the error $u_{h}\left(t_{n}\right)-u_{h}^{n}$ in the case of $\alpha=1$, we employ a parabolic argument that has already been used in 23 . for the CrankNicolson scheme of the time-dependent Navier-Stokes equation. Let $1 \leq m \leq N$ be given. We consider the linearized "backward" counterpart of the discrete NavierStokes (4.1): For $\xi^{n} \in V_{h}, 1 \leq n \leq m$, find $\Phi_{h}^{n-1} \in V_{h}$ such that

$$
\left(v_{h}, d_{t} \Phi_{h}^{n}\right)-a\left(v_{h}, \Phi_{h}^{n-1}\right)-b\left(u_{h}^{n}, v_{h}, \Phi_{h}^{n-1}\right)-b\left(v_{h}, u_{h}^{n}, \Phi_{h}^{n-1}\right)=\left(v_{h}, \xi^{n}\right),
$$

for $v_{h} \in V_{h}$ with an initial value $\Phi_{h}^{m}=0$.

Here, we need to introduce the following discrete dual Gronwall lemma provided in [11].

Lemma 5.1. Let $C>0$ and let $a_{n}, b_{n}, d_{n}$, for integers $0 \leq n \leq m$, be nonnegative numbers such that

$$
a_{k}+\tau \sum_{n=k}^{m} b_{n} \leq \tau \sum_{n=k+1}^{m} d_{n} a_{n}+C, 0 \leq k \leq m .
$$

Then

$$
a_{k}+\tau \sum_{n=k}^{m} b_{n} \leq C \exp \left(\tau \sum_{n=k+1}^{m} d_{n}\right), 0 \leq k \leq m,
$$

where we assume that $\tau \sum_{n=m+1}^{m} d_{n}=0$.

The following lemma provides the stability of the scheme (5.1).

Lemma 5.2. Under the assumptions of Theorem 4.1, the following a priori estimate holds:

$$
\left\|\Phi_{h}^{k}\right\|_{1}^{2}+\nu \tau \sum_{n=k}^{m}\left\|A_{h} \Phi_{h}^{n}\right\|_{0}^{2} \leq \kappa \tau \sum_{n=1}^{m}\left\|\xi^{n}\right\|_{0}^{2},
$$

for all $0 \leq k \leq m$.

Proof. The proof follows the line of argument used in the proofs of Theorem 4.1, In view of Lemma 3.1 and (4.3), we can prove that (5.1) admits a unique solution sequence $\left\{\Phi_{h}^{k}\right\}_{0}^{m}$. 
Moreover, by taking $v_{h}=-2 A_{h} \Phi_{h}^{n-1} \tau$ in (5.1), we obtain

$$
\begin{aligned}
\left\|\Phi_{h}^{n-1}\right\|_{1}^{2} & -\left\|\Phi_{h}^{n}\right\|_{1}^{2}+\left\|d_{t} \Phi_{h}^{n}\right\|_{1}^{2} \tau^{2}+2 \nu\left\|A_{h} \Phi_{h}^{n-1}\right\|_{0}^{2} \tau \\
& +2 b\left(A_{h} \Phi_{h}^{n-1}, u_{h}^{n}, \Phi_{h}^{n-1}\right) \tau+2 b\left(u_{h}^{n}, A_{h} \Phi_{h}^{n-1}, \Phi_{h}^{n-1}\right) \tau \\
& \leq \frac{\nu}{4}\left\|A_{h} \Phi_{h}^{n-1}\right\|_{0}^{2} \tau+\frac{4}{\nu}\left\|\xi^{n}\right\|_{0}^{2} \tau .
\end{aligned}
$$

From Lemma 3.1 and (3.10), we have

$$
\begin{aligned}
2\left|b\left(A_{h} \Phi_{h}^{n-1}, u_{h}^{n}, \Phi_{h}^{n}\right)\right| \tau & +2\left|b\left(u_{h}^{n}, A_{h} \Phi_{h}^{n-1}, \Phi_{h}^{n}\right)\right| \tau \\
& \leq 2 c_{0} \gamma_{0}\left\|A_{h} u_{h}^{n}\right\|_{0}\left\|\Phi_{h}^{n}\right\|_{1}\left\|A_{h} \Phi_{h}^{n-1}\right\|_{0} \tau \\
& \leq \frac{\nu}{4}\left\|A_{h} \Phi_{h}^{n-1}\right\|_{0}^{2} \tau+4 \nu^{-1} c_{0}^{2} \gamma_{0}^{2}\left\|A_{h} u_{h}^{n}\right\|_{0}^{2}\left\|\Phi_{h}^{n}\right\|_{1}^{2} \tau \\
2\left|b\left(A_{h} \Phi_{h}^{n-1}, u_{h}^{n}, \Phi_{h}^{n}-\Phi_{h}^{n-1}\right)\right| \tau & +2\left|b\left(u_{h}^{n}, A_{h} \Phi_{h}^{n-1}, \Phi_{h}^{n}-\Phi_{h}^{n-1}\right)\right| \tau \\
& \leq \frac{1}{2} G^{1 / 2}\left(u_{h}^{n}\right)\left\|d_{t} \Phi_{h}^{n}\right\|_{1}\left\|A_{h} \Phi_{h}^{n-1}\right\|_{0} \tau^{2} \\
& \leq \frac{1}{2}\left\|d_{t} \Phi_{h}^{n}\right\|_{1}^{2} \tau^{2}+\frac{1}{4} G\left(u_{h}^{n}\right)\left\|A_{h} \Phi_{h}^{n-1}\right\|_{0}^{2} \tau^{2} .
\end{aligned}
$$

Combining (5.5) with the above estimate gives

$$
\begin{aligned}
\left\|\Phi_{h}^{n-1}\right\|_{1}^{2} & -\left\|\Phi_{h}^{n}\right\|_{1}^{2}+\nu\left\|A_{h} \Phi_{h}^{n-1}\right\|_{0}^{2} \tau+\frac{1}{4}\left(\nu-G\left(u_{h}^{n}\right) \tau\right)\left\|A_{h} \Phi_{h}^{n-1}\right\|_{0}^{2} \tau \\
& \leq 4 \nu^{-1} c_{0}^{2} \gamma_{0}^{2}\left\|A_{h} u_{h}^{n}\right\|_{0}^{2}\left\|\Phi_{h}^{n}\right\|_{1}^{2} \tau+4 \nu^{-1}\left\|\xi^{n}\right\|_{0}^{2} \tau,
\end{aligned}
$$

for all $1 \leq n \leq m$. Using (4.3) and Theorem 4.1 with $\alpha=1,2$, we have

$$
\nu-G\left(u_{h}^{n}\right) \tau \geq \nu-G_{h} \tau \geq 0, \forall 0 \leq n \leq N .
$$

Summing (5.6) from $k+1$ to $m$ and using (5.7) and Theorem 4.1, we obtain

$$
\begin{aligned}
\left\|\Phi_{h}^{k}\right\|_{1}^{2} & +\nu \tau \sum_{n=k}^{m-1}\left\|A_{h} \Phi_{h}^{n}\right\|_{0}^{2} \\
& \leq 4 \tau \sum_{n=k+1}^{m} \nu^{-1} c_{0}^{2} \gamma_{0}^{2}\left\|A_{h} u_{h}^{n}\right\|_{0}^{2}\left\|\Phi_{h}^{n}\right\|_{1}^{2}+4 \nu^{-1} \tau \sum_{n=1}^{m}\left\|\xi^{n}\right\|_{0}^{2}
\end{aligned}
$$

for all $0 \leq k \leq m-1$. Applying Lemma 5.1 to (5.8) and using Theorem 4.1 yields (5.4).

\section{ERROR ANALYSIS}

In this section, we establish the $H^{1}$ - and $L^{2}$-bounds of the error $e^{n}=u_{h}\left(t_{n}\right)-u_{h}^{n}$ and the $L^{2}$-bound of the error $\eta^{n}=p_{h}\left(t_{n}\right)-p_{h}^{n}$ for all $1 \leq n \leq N$. To do this, we take $t=t_{n}$ in (3.8) and note

$$
u_{h t}\left(t_{n}\right)-d_{t} u_{h}\left(t_{n}\right)=\tau^{-1} \int_{t_{n-1}}^{t_{n}}\left(u_{h t}\left(t_{n}\right)-u_{h t}(t)\right) d t=\tau^{-1} \int_{t_{n-1}}^{t_{n}}\left(t-t_{n-1}\right) u_{h t t} d t
$$

to obtain

$$
\begin{aligned}
\left(d_{t} u_{h}\left(t_{n}\right), v_{h}\right)+ & a\left(u_{h}\left(t_{n}\right), v_{h}\right)-d\left(v_{h}, p_{h}\left(t_{n}\right)\right)+d\left(u_{h}\left(t_{n}\right), q_{h}\right)+b\left(u_{h}\left(t_{n}\right), u_{h}\left(t_{n}\right), v_{h}\right) \\
(6.1) \quad= & \left(f\left(t_{n}\right), v_{h}\right)-\frac{1}{\tau} \int_{t_{n-1}}^{t_{n}}\left(t-t_{n-1}\right)\left(u_{h t t}(t), v_{h}\right) d t
\end{aligned}
$$


Subtracting (4.1) from (6.1), we obtain

$$
\begin{aligned}
\left(d_{t} e^{n}, v_{h}\right) & +a\left(e^{n}, v_{h}\right)-d\left(v_{h}, \eta^{n}\right)+d\left(e^{n}, q_{h}\right)+b\left(e^{n}, u_{h}\left(t_{n}\right), v_{h}\right) \\
& +b\left(u_{h}^{n}, e^{n}, v_{h}\right)=\left(E_{n}, v_{h}\right),
\end{aligned}
$$

for all $\left(v_{h}, q_{h}\right) \in\left(X_{h}, M_{h}\right)$ with

$$
\begin{aligned}
\left(E_{n}, v_{h}\right) & =-\frac{1}{\tau} \int_{t_{n-1}}^{t_{n}}\left(t-t_{n-1}\right)\left(u_{h t t}(t), v_{h}\right) d t \\
& +b\left(u_{h}^{n-1}-u_{h}^{n}, u_{h}^{n-1}, v_{h}\right)+b\left(u_{h}^{n}, u_{h}^{n-1}-u_{h}^{n}, v_{h}\right) .
\end{aligned}
$$

Lemma 6.1. Under the assumptions of Theorem 4.1 with $\alpha=1,2$, the error $E_{n}$ satisfies the following bounds:

$$
\begin{aligned}
& \tau \sum_{n=1}^{m}\left\|A_{h}^{-1} P_{h} E_{n}\right\|_{0}^{2} \leq \kappa \tau^{2}, \\
& \tau \sum_{n=1}^{m}\left\|A_{h}^{-1 / 2} P_{h} E_{h}\right\|_{0}^{2} \leq \kappa \tau^{\alpha}, \\
& \tau \sum_{n=1}^{m} \sigma^{2-\alpha}\left(t_{n}\right)\left\|A_{h}^{-1 / 2} P_{h} E_{h}\right\|_{0}^{2} \leq \kappa \tau^{2},
\end{aligned}
$$

for all $1 \leq m \leq N$, and

$$
\begin{array}{r}
\sigma^{4-\alpha}\left(t_{m}\right)\left\|E_{m}\right\|_{0}^{2}+\tau \sum_{n=2}^{m} \sigma^{3-\alpha}\left(t_{n}\right)\left\|E_{n}\right\|_{0}^{2} \leq \kappa \tau^{2}, 2 \leq m \leq N, \\
\tau \sum_{n=3}^{m} \sigma^{4-\alpha}\left(t_{n}\right)\left\|A_{h}^{-1 / 2} P_{h} d_{t} E_{n}\right\|_{0}^{2} \leq \kappa \tau^{2}, 3 \leq m \leq N .
\end{array}
$$

Proof. First, it follows from (6.3), (3.10) and Lemma 3.1 that

$$
\begin{aligned}
\left\|A_{h}^{-1} P_{h} E_{n}\right\|_{0}^{2} \tau & \leq c \tau^{2} \int_{t_{n-1}}^{t_{n}}\left\|A_{h}^{-1} u_{h t t}\right\|_{0}^{2} d t \\
& +c\left\|d_{t} u_{h}^{n}\right\|_{0}^{2}\left(\left\|u_{h}^{n}\right\|_{1}^{2}+\left\|u_{h}^{n-1}\right\|_{1}^{2}\right) \tau^{3} \\
\left\|A_{h}^{-1 / 2} P_{h} E_{n}\right\|_{0}^{2} \tau & \leq c \tau^{\alpha} \int_{t_{n-1}}^{t_{n}} \sigma^{2-\alpha}(t)\left\|A_{h}^{-1 / 2} u_{h t t}\right\|_{0}^{2} d t \\
& +c\left\|d_{t} u_{h}^{n}\right\|_{1}^{2}\left(\left\|u_{h}^{n-1}\right\|_{1}^{2}+\left\|u_{h}^{n}\right\|_{1}^{2}\right) \tau^{3}, \\
\sigma^{2-\alpha}\left(t_{n}\right)\left\|A_{h}^{-1 / 2} P_{h} E_{n}\right\|_{0}^{2} \tau & \leq c \tau^{2} \int_{t_{n-1}}^{t_{n}} \sigma^{2-\alpha}(t)\left\|A_{h}^{-1 / 2} u_{h t t}\right\|_{0}^{2} d t \\
& +c \sigma^{2-\alpha}\left(t_{n}\right)\left\|d_{t} u_{h}^{n}\right\|_{1}^{2}\left(\left\|u_{h}^{n-1}\right\|_{1}^{2}+\left\|u_{h}^{n}\right\|_{1}^{2}\right) \tau^{3} .
\end{aligned}
$$

Summing (6.9), (6.10) and (6.11) from 1 to $m$, respectively, noting $\tau^{2} \leq \sigma^{2-\alpha}\left(t_{n}\right) \tau^{\alpha}$ and using (2.2), Theorem 3.6 and Theorem 4.1, we deduce (6.4)-(6.6) for $\alpha=1,2$.

Next, by using (3.10) and Lemma 3.1, we deduce from (6.3) that

$$
\begin{aligned}
\left\|E_{n}\right\|_{0} & \leq c \tau^{-1 / 2}\left(\int_{t_{n-1}}^{t_{n}}\left(t-t_{n-1}\right)^{2}\left\|u_{h t t}\right\|_{0}^{2} d t\right)^{1 / 2} \\
& +c\left(\left\|A_{h} u_{h}^{n}\right\|_{0}+\left\|A_{h} u^{n-1}\right\|_{0}\right)\left\|d_{t} u_{h}^{n}\right\|_{0} \tau,
\end{aligned}
$$


for all $2 \leq n \leq N$. Hence, we deduce from (6.12) that

$$
\begin{aligned}
\sigma^{3-\alpha}\left(t_{n}\right)\left\|E_{n}\right\|_{0}^{2} \tau & \leq c \tau^{2} \int_{t_{n-1}}^{t_{n}} \sigma^{3-\alpha}(t)\left\|u_{h t t}\right\|_{0}^{2} d t \\
(6.13) & +c\left(\sigma\left(t_{n}\right)\left\|A_{h} u_{h}^{n}\right\|_{0}^{2}+\sigma\left(t_{n-1}\right)\left\|A_{h} u^{n-1}\right\|_{0}^{2}\right) \sigma^{2-\alpha}\left(t_{n}\right)\left\|d_{t} u_{h}^{n}\right\|_{1}^{2} \tau^{3}, \\
\sigma^{4-\alpha}\left(t_{n}\right)\left\|E_{n}\right\|_{0}^{2} & \leq c \tau^{2} \int_{t_{n-1}}^{t_{n}} \sigma^{3-\alpha}(t)\left\|u_{h t t}\right\|_{0}^{2} d t \\
(6.14) & +c\left(\sigma\left(t_{n}\right)\left\|A_{h} u_{h}^{n}\right\|_{0}^{2}+\sigma\left(t_{n-1}\right)\left\|A_{h} u^{n-1}\right\|_{0}^{2}\right) \sigma^{3-\alpha}\left(t_{n}\right)\left\|d_{t} u_{h}^{n}\right\|_{1}^{2} \tau^{2},
\end{aligned}
$$

for all $2 \leq n \leq N$. Summing (6.13) from $n=2$ to $n=m$ and using (6.14) and Theorems 3.6, 4.1 and 4.2, we deduce (6.7).

Moreover, we deduce from (6.3) that

$$
\begin{aligned}
\left(d_{t} E_{n}, v_{h}\right) & =-\tau^{-2} \int_{t_{n-1}}^{t_{n}}\left(t-t_{n-1}\right) \int_{t-\tau}^{t}\left(u_{h t t t}(s), v_{h}\right) d s d t \\
& -b\left(d_{t t} u_{h}^{n}, u_{h}^{n-1}, v_{h}\right) \tau-b\left(u_{h}^{n-1}, d_{t t} u_{h}^{n}, v_{h}\right) \tau \\
& -b\left(d_{t} u_{h}^{n}, d_{t} u_{h}^{n}, v_{h}\right) \tau-b\left(d_{t} u_{h}^{n-1}, d_{t} u_{h}^{n-1}, v_{h}\right) \tau
\end{aligned}
$$

for all $3 \leq n \leq N$. Using (3.10) and Lemma 3.1, we deduce from (6.15) that

$$
\begin{aligned}
\left\|A_{h}^{-1 / 2} P_{h} d_{t} E_{n}\right\|_{0} & \leq c \tau^{-3 / 2}\left(\int_{t_{n-1}}^{t_{n}}\left(t-t_{n-1}\right)^{2}\left\|\int_{t-\tau}^{t} u_{h t t t}(s) d s\right\|_{-1}^{2} d t\right)^{1 / 2} \\
& +c\left\|d_{t t} u_{h}^{n}\right\|_{0}\left\|A_{h} u_{h}^{n-1}\right\|_{0} \tau+c\left\|d_{t} u_{h}^{n}\right\|_{0}\left\|A_{h} d_{t} u_{h}^{n}\right\|_{0} \tau \\
& +c\left\|d_{t} u_{h}^{n-1}\right\|_{0}\left\|A_{h} d_{t} u_{h}^{n-1}\right\|_{0} \tau
\end{aligned}
$$

which yields

$$
\begin{aligned}
\sigma^{4-\alpha}\left(t_{n}\right)\left\|A_{h}^{-1 / 2} P_{h} d_{t} E_{n}\right\|_{0}^{2} \tau & \leq c \tau^{2} \int_{t_{n-2}}^{t_{n}} \sigma^{4-\alpha}(t)\left\|A_{h}^{-1 / 2} u_{h t t t}\right\|_{0}^{2} d t \\
& +c \tau^{3} \sigma^{3-\alpha}\left(t_{n}\right) \sigma\left(t_{n-1}\right)\left\|d_{t t} u_{h}^{n}\right\|_{0}^{2}\left\|A_{h} u_{h}^{n-1}\right\|_{0}^{2} \\
& +c \tau^{3} \sigma^{4-\alpha}\left(t_{n}\right)\left\|d_{t} u_{h}^{n}\right\|_{0}^{2}\left\|A_{h} d_{t} u_{h}^{n}\right\|_{0}^{2} \\
& +c \tau^{3} \sigma^{4-\alpha}\left(t_{n-1}\right)\left\|d_{t} u_{h}^{n-1}\right\|_{0}^{2}\left\|A_{h} d_{t} u_{h}^{n-1}\right\|_{0}^{2},
\end{aligned}
$$

for all $3 \leq n \leq N$. Summing (6.16) from 3 to $m$ and using Theorems 3.6, 4.1 and 4.2 , we deduce (6.8).

Lemma 6.2. Under the assumptions of Theorem 4.1 with $\alpha=1,2$, we have

$$
\left\|e^{m}\right\|_{0}^{2}+\tau \sum_{n=1}^{m}\left(\left\|d_{t} e^{n}\right\|_{0}^{2} \tau+\nu\left\|e^{n}\right\|_{1}^{2}\right) \leq \kappa \tau^{\alpha},
$$

for all $1 \leq m \leq N$

Proof. Taking $v_{h}=2 e^{n} \tau \in V_{h}$ and $q_{h}=0$ in (6.2), we obtain

$$
\begin{aligned}
\left\|e^{n}\right\|_{0}^{2} & -\left\|e^{n-1}\right\|_{0}^{2}+\left\|d_{t} e^{n}\right\|_{0}^{2} \tau^{2}+2 \nu\left\|e^{n}\right\|_{1}^{2} \tau+2 b\left(e^{n}, u_{h}^{n}, e^{n}\right) \tau \\
& \leq \frac{\nu}{4}\left\|e^{n}\right\|_{1}^{2} \tau+4 \nu^{-1}\left\|A_{h}^{-1 / 2} P_{h} E_{n}\right\|_{0}^{2} \tau .
\end{aligned}
$$


Using Lemma 3.1 and (3.10), one finds

$$
\begin{aligned}
2\left|b\left(e^{n-1}, u_{h}^{n}, e^{n}\right)\right| \tau & \leq 2 c_{0} \gamma_{0}^{1 / 2}\left\|e^{n-1}\right\|_{0}^{1 / 2}\left\|e_{h}^{n-1}\right\|_{1}^{1 / 2}\left\|u_{h}^{n}\right\|_{1}\left\|e^{n}\right\|_{1} \tau \\
& \leq \frac{\nu}{4}\left(\left\|e^{n}\right\|_{1}^{2}+\left\|e^{n-1}\right\|_{1}^{2}\right) \tau+2\left(\frac{2}{\nu}\right)^{3} c_{0}^{4} \gamma_{0}^{2}\left\|u_{h}^{n}\right\|_{1}^{4}\left\|e^{n-1}\right\|_{0}^{2} \tau, \\
2\left|b\left(e^{n}-e^{n-1}, u_{h}^{n}, e^{n}\right)\right| \tau & \leq \frac{1}{2} G^{1 / 2}\left(u_{h}^{n}\right)\left\|e^{n}\right\|_{1}\left\|d_{t} e^{n}\right\|_{0} \tau^{2} \\
& \leq \frac{1}{2}\left\|d_{t} e^{n}\right\|_{0}^{2} \tau^{2}+\frac{1}{4} G\left(u_{h}^{n}\right)\left\|e^{n}\right\|_{1}^{2} \tau^{2} .
\end{aligned}
$$

Hence, by combining the above inequalities with (6.18), we obtain

$$
\begin{aligned}
\left\|e^{n}\right\|_{0}^{2} & -\left\|e^{n-1}\right\|_{0}^{2}+\frac{1}{2}\left\|d_{t} e^{n}\right\|_{0}^{2} \tau^{2}+\nu\left\|e^{n}\right\|_{1}^{2} \tau \\
& +\frac{\nu}{4}\left(\left\|e^{n}\right\|_{1}^{2}-\left\|e^{n-1}\right\|_{1}^{2}\right) \tau+\frac{1}{4}\left(\nu-G\left(u_{h}^{n}\right) \tau\right)\left\|e^{n}\right\|_{1}^{2} \tau \\
& \leq 2\left(\frac{2}{\nu}\right)^{3} c_{0}^{4} \gamma_{0}^{2}\left\|u_{h}^{n}\right\|_{1}^{4}\left\|e^{n-1}\right\|_{0}^{2} \tau+4 \nu^{-1}\left\|A_{h}^{-1 / 2} P_{h} E_{n}\right\|_{0}^{2} \tau
\end{aligned}
$$

for all $1 \leq n \leq N$. Moreover, summing (6.19) from 1 to $m$ and using (5.7), we have

$$
\begin{aligned}
\left\|e^{m}\right\|_{0}^{2} & +\tau \sum_{n=1}^{m}\left(\frac{1}{2}\left\|d_{t} e^{n}\right\|_{0}^{2} \tau+\nu\left\|e^{n}\right\|_{1}^{2}\right) \\
& \leq \tau \sum_{n=0}^{m-1} d_{n}\left\|e^{n}\right\|_{0}^{2}+4 \nu^{-1} \tau \sum_{n=1}^{N}\left\|A_{h}^{-1 / 2} P_{h} E_{n}\right\|_{0}^{2},
\end{aligned}
$$

where $d_{n}=2\left(\frac{2}{\nu}\right)^{3} c_{0}^{4} \gamma_{0}^{2}\left\|u_{h}^{n+1}\right\|_{1}^{4}$. We set

$$
a_{n}=\left\|e^{n}\right\|_{0}^{2}, b_{n}=\frac{1}{2}\left\|d_{t} e^{n}\right\|_{0}^{2} \tau+\nu\left\|e^{n}\right\|_{1}^{2}, C=4 \nu^{-1} \tau \sum_{n=1}^{N}\left\|A_{h}^{-1 / 2} P_{h} E_{n}\right\|_{0}^{2},
$$

and apply Lemma 3.5 to (6.20) and use Theorem 4.1 and Lemma 6.1 to deduce (6.17).

With the aid of Lemma 6.2, we obtain the following error estimate.

Lemma 6.3. Under the assumptions of Theorem 4.1 with $\alpha=1,2$, we have

$$
\sigma^{2-\alpha}\left(t_{m}\right)\left\|e^{m}\right\|_{0}^{2}+\tau \sum_{n=1}^{m} \sigma^{2-\alpha}\left(t_{n}\right)\left\|e^{n}\right\|_{1}^{2} \leq \kappa \tau^{2},
$$

for all $1 \leq m \leq N$.

Proof. For $\alpha=2$, Lemma 6.2 yields (6.21). For $\alpha=1$, we let $\left\{\Phi_{h}^{n}\right\}_{0}^{m}$ be the solution of (5.1), corresponding to the initial value $\Phi_{h}^{m}=0$ and the right-hand side of $\left\{\xi^{n}\right\}_{1}^{m}=\left\{e^{n}\right\}_{1}^{m}$. Then, by construction, it holds that

$$
\begin{aligned}
\left\|e^{n}\right\|_{0}^{2} \tau & =\left(e^{n}, d_{t} \Phi_{h}^{n}\right) \tau-a\left(e^{n}, \Phi_{h}^{n-1}\right) \tau \\
& -b\left(u_{h}^{n}, e^{n}, \Phi_{h}^{n-1}\right) \tau-b\left(e^{n}, u_{h}^{n}, \Phi_{h}^{n-1}\right) \tau .
\end{aligned}
$$

Taking $v_{h}=\Phi_{h}^{n-1} \tau$ in (6.2) and adding (6.22), we obtain

$$
\left\|e^{n}\right\|_{0}^{2} \tau=\left(e^{n}, \Phi_{h}^{n}\right)-\left(e^{n-1}, \Phi_{h}^{n-1}\right)-\left(E_{n}, \Phi_{h}^{n-1}\right) \tau+b\left(e^{n}, e^{n}, \Phi_{h}^{n-1}\right) \tau .
$$


Summing (6.23) for $1 \leq n \leq m$ and using Lemmas 5.2, 6.1 and 6.2, we have

$$
\begin{aligned}
\tau \sum_{n=1}^{m}\left\|e^{n}\right\|_{0}^{2} & \leq\left(\tau \sum_{n=1}^{m}\left\|A_{h}^{-1} P_{h} E_{n}\right\|_{0}^{2}\right)^{1 / 2}\left(\tau \sum_{n=1}^{m}\left\|A_{h} \Phi_{h}^{n-1}\right\|_{0}^{2}\right)^{1 / 2} \\
& +c\left(\tau \sum_{n=1}^{m}\left\|e^{n}\right\|_{0}^{2}\left\|e^{n}\right\|_{1}^{2}\right)^{1 / 2}\left(\tau \sum_{n=1}^{m}\left\|A_{h} \Phi_{h}^{n-1}\right\|_{0}^{2}\right)^{1 / 2} \\
& \leq \kappa \tau\left(\tau \sum_{n=1}^{m}\left\|e^{n}\right\|_{0}^{2}\right)^{1 / 2} \leq \frac{1}{2} \tau \sum_{n=1}^{m}\left\|e^{n}\right\|_{0}^{2}+\kappa \tau^{2} .
\end{aligned}
$$

Next, multiplying (6.19) by $\sigma\left(t_{n}\right)$, we deduce

$$
\begin{aligned}
\sigma\left(t_{n}\right)\left\|e^{n}\right\|_{0}^{2} & -\sigma\left(t_{n-1}\right)\left\|e^{n-1}\right\|_{0}^{2}+\sigma\left(t_{n}\right) \nu\left\|e^{n}\right\|_{1}^{2} \tau+\frac{\nu}{4}\left(\sigma\left(t_{n}\right)\left\|e^{n}\right\|_{1}^{2}-\sigma\left(t_{n-1}\right)\left\|e^{n-1}\right\|_{1}^{2}\right) \tau \\
& \leq\left\|e^{n-1}\right\|_{0}^{2} \tau+\frac{\nu}{4}\left\|e_{h}^{n-1}\right\|_{1}^{2} \tau^{2}+2\left(\frac{2}{\nu}\right)^{3} c_{0}^{4} \gamma_{0}^{2}\left\|u_{h}^{n}\right\|_{1}^{4}\left\|e^{n-1}\right\|_{0}^{2} \tau \\
& +4 \nu^{-1} \sigma\left(t_{n}\right)\left\|A_{h}^{-1 / 2} P_{h} E_{n}\right\|_{0}^{2} \tau,
\end{aligned}
$$

for all $1 \leq n \leq N$. Summing (6.25) from $n=1$ to $n=m$, we have

$$
\begin{aligned}
\sigma\left(t_{m}\right)\left\|e^{m}\right\|_{0}^{2} & +\nu \tau \sum_{n=1}^{m} \sigma\left(t_{n}\right)\left\|e^{n}\right\|_{1}^{2}+\frac{\nu}{4} \sigma\left(t_{m}\right)\left\|e^{m}\right\|_{1}^{2} \tau \\
& \leq \tau \sum_{n=1}^{m}\left(\left\|e^{n}\right\|_{0}^{2}+\tau \nu\left\|e^{n}\right\|_{1}^{2}\right)+2 \tau \sum_{n=1}^{m}\left(\frac{2}{\nu}\right)^{3} c_{0}^{4} \gamma_{0}^{2}\left\|u_{h}^{n}\right\|_{1}^{4}\left\|e^{n-1}\right\|_{0}^{2} \\
& +4 \tau \sum_{n=1}^{m} \nu^{-1} \sigma\left(t_{n}\right)\left\|A_{h}^{-1 / 2} P_{h} E_{n}\right\|_{0}^{2} .
\end{aligned}
$$

Using (6.24), Theorem 4.1 and Lemmas 6.1 and 6.2 in the above inequality gives (6.21) for $\alpha=1$.

Lemma 6.4. Under the assumptions of Theorem 4.1 with $\alpha=1,2$, we have

$$
\sigma^{3-\alpha}\left(t_{m}\right)\left\|e^{m}\right\|_{1}^{2}+\tau \sum_{n=2}^{m} \sigma^{3-\alpha}\left(t_{n}\right)\left(\left\|d_{t} e^{n}\right\|_{0}^{2}+\nu^{2}\left\|A_{h} e^{n}\right\|_{0}^{2}\right) \leq \kappa \tau^{2}
$$

for all $1 \leq m \leq N$.

Proof. Taking $v_{h}=2 A_{h} e^{n} \tau \in V_{h}$ and $q_{h}=0$ in (6.2), we obtain

$$
\begin{aligned}
\left\|e^{n}\right\|_{1}^{2} & -\left\|e^{n-1}\right\|_{1}^{2}+\left\|d_{t} e^{n}\right\|_{1}^{2} \tau^{2}+2 \nu\left\|A_{h} e^{n}\right\|_{0}^{2} \tau+2 b\left(e^{n}, u_{h}\left(t_{n}\right), A_{h} e^{n}\right) \tau \\
& +2 b\left(u_{h}^{n}, e_{h}^{n}, A_{h} e^{n}\right) \tau \leq \frac{\nu}{4}\left\|A_{h} e^{n}\right\|_{0}^{2} \tau+4 \nu^{-1}\left\|E_{n}\right\|_{0}^{2} \tau .
\end{aligned}
$$

In view of Lemma 3.1 and (3.10), we have

$$
\begin{aligned}
2\left|b\left(e^{n}, u_{h}\left(t_{n}\right), e^{n}\right)\right| \tau & +2\left|b\left(u_{h}^{n}, e^{n}, A_{h} e^{n}\right)\right| \tau \\
& \leq 2 c_{0} \gamma_{0}^{1 / 2}\left\|e^{n}\right\|_{1}\left(\left\|A_{h} u_{h}^{n}\right\|_{0}+\left\|A_{h} u_{h}\left(t_{n}\right)\right\|_{0}\right)\left\|A_{h} e^{n}\right\|_{0} \tau \\
& \leq \frac{\nu}{4}\left\|A_{h} e^{n}\right\|_{0}^{2} \tau+c\left(\left\|A_{h} u_{h}\left(t_{n}\right)\right\|_{0}^{2}+\left\|A_{h} u_{h}^{n}\right\|_{0}^{2}\right)\left\|e_{h}^{n}\right\|_{1}^{2} \tau .
\end{aligned}
$$

Hence, by combining the above inequality with (6.27), we obtain

$$
\begin{aligned}
\left\|e^{n}\right\|_{1}^{2} & -\left\|e^{n-1}\right\|_{1}^{2}+\nu\left\|A_{h} e^{n}\right\|_{0}^{2} \tau \\
& \leq c\left(\left\|A_{h} u_{h}^{n}\right\|_{0}^{2}+\left\|A_{h} u_{h}\left(t_{n}\right)\right\|_{0}^{2}\right)\left\|e^{n}\right\|_{1}^{2} \tau+4 \nu^{-1}\left\|E_{n}\right\|_{0}^{2} \tau,
\end{aligned}
$$


for all $1 \leq n \leq N$. Multiplying (6.28) by $\sigma^{3-\alpha}\left(t_{n}\right)$, we find

$$
\begin{aligned}
\sigma^{3-\alpha}\left(t_{n}\right)\left\|e^{n}\right\|_{1}^{2} & -\sigma^{3-\alpha}\left(t_{n-1}\right)\left\|e^{n-1}\right\|_{1}^{2}+\nu \sigma^{3-\alpha}\left(t_{n}\right)\left\|A_{h} e^{n}\right\|_{0}^{2} \tau \\
& \leq c \sigma^{3-\alpha}\left(t_{n}\right)\left(\left\|A_{h} u_{h}^{n}\right\|_{0}^{2}+\left\|A_{h} u_{h}\left(t_{n}\right)\right\|_{0}^{2}\right)\left\|e^{n}\right\|_{1}^{2} \tau \\
& +c \sigma^{2-\alpha}\left(t_{n-1}\right)\left\|e^{n-1}\right\|_{1}^{2} \tau+4 \nu^{-1} \sigma^{3-\alpha}\left(t_{n}\right)\left\|E_{n}\right\|_{0}^{2} \tau
\end{aligned}
$$

for all $1 \leq n \leq N$. Summing (6.29) from 2 to $m$, and using Theorems 3.3 and 4.1 and Lemmas 6.1, 6.2 and 6.3, we deduce

$$
\sigma^{3-\alpha}\left(t_{m}\right)\left\|e^{m}\right\|_{1}^{2}+\nu \tau \sum_{n=2}^{m} \sigma^{3-\alpha}\left(t_{n}\right)\left\|A_{h} e^{n}\right\|_{0}^{2} \leq \kappa \tau^{2},
$$

for all $1 \leq m \leq N$.

Finally, we deduce from (6.2), (3.10) and Lemma 3.1 that

$$
\begin{aligned}
\sigma^{3-\alpha}\left(t_{n}\right)\left\|d_{t} e^{n}\right\|_{0}^{2} & \leq c \sigma^{3-\alpha}\left(t_{n}\right)\left(1+\left\|u_{h}^{n}\right\|_{1}^{2}+\left\|e_{h}^{n}\right\|_{1}^{2}\right)\left\|A_{h} e^{n}\right\|_{0}^{2} \tau \\
& +c \sigma^{3-\alpha}\left(t_{n}\right)\left\|E_{n}\right\|_{0}^{2} \tau,
\end{aligned}
$$

for all $2 \leq n \leq N$. Summing (6.31) from $n=2$ to $n=m$ and using (6.30), Theorem 4.1 and Lemmas 6.1 and 6.2, we deduce (6.26).

It remains to prove the error estimate for the discrete pressure $p_{h}^{m}$. To do this, we need to estimate $d_{t} e^{n}$. It follows from (6.2) that

$$
\begin{aligned}
\left(d_{t t} e^{n}, v_{h}\right) & +a\left(d_{t} e^{n}, v_{h}\right)+b\left(d_{t} e^{n}, u_{h}\left(t_{n}\right), v_{h}\right)+b\left(e^{n-1}, d_{t} u_{h}\left(t_{n}\right), v_{h}\right) \\
& +b\left(d_{t} u_{h}^{n}, e^{n}, v_{h}\right)+b\left(u_{h}^{n-1}, d_{t} e^{n}, v_{h}\right)=\left(d_{t} E_{n}, v_{h}\right),
\end{aligned}
$$

for all $v_{h} \in V_{h}$ and $1 \leq n \leq N$. Taking $v_{h}=2 d_{t} e^{n} \tau$ in (6.32) and using (3.12), we get

$$
\begin{aligned}
\left\|d_{t} e^{n}\right\|_{0}^{2} & -\left\|d_{t} e^{n-1}\right\|_{0}^{2}+2 \nu\left\|d_{t} e^{n}\right\|_{1}^{2} \tau+2 b\left(d_{t} e^{n}, u_{h}\left(t_{n}\right), d_{t} e^{n}\right) \tau \\
& +2 b\left(e^{n-1}, d_{t} u_{h}^{n}, d_{t} e^{n}\right) \tau \\
& +2 b\left(d_{t} u_{h}^{n}, e^{n}, d_{t} e^{n}\right) \tau \leq \frac{\nu}{4}\left\|d_{t} e^{n}\right\|_{1}^{2} \tau+4 \nu^{-1}\left\|A_{h}^{-1 / 2} P_{h} d_{t} E_{n}\right\|_{0}^{2} \tau .
\end{aligned}
$$

In view of (3.10) and Lemma 3.1, we deduce

$$
\begin{aligned}
2\left|b\left(d_{t} e^{n}, u_{h}\left(t_{n}\right), d_{t} e^{n}\right)\right| \tau & \leq c_{0} \gamma_{0}^{1 / 2}\left\|d_{t} e^{n}\right\|_{0}^{1 / 2}\left\|d_{t} e^{n}\right\|_{1}^{3 / 2}\left\|u_{h}\left(t_{n}\right)\right\|_{1} \tau \\
& \leq \frac{\nu}{4}\left\|d_{t} e^{n}\right\|_{1}^{2} \tau+\left(\frac{2}{\nu}\right)^{3} c_{0}^{4} \gamma_{0}^{2}\left\|u_{h}\left(t_{n}\right)\right\|_{1}^{4}\left\|d_{t} e^{n}\right\|_{0}^{2} \tau, \\
2\left|b\left(e^{n-1}, d_{t} u_{h}^{n}, d_{t} e^{n}\right)\right| \tau & +2 b\left(d_{t} u_{h}^{n}, e^{n}, d_{t} e^{n}\right) \tau \\
& \leq 2 c_{0} \gamma_{0}\left(\left\|A_{h} e^{n-1}\right\|_{0}+\left\|A_{h} e^{n}\right\|_{0}\right)\left\|d_{t} u_{h}^{n}\right\|_{0}\left\|d_{t} e^{n}\right\|_{1} \tau \\
& \leq \frac{\nu}{4}\left\|d_{t} e^{n}\right\|_{1}^{2} \tau+8 \nu^{-1} c_{0}^{2} \gamma_{0}^{2}\left(\left\|A_{h} e^{n-1}\right\|_{0}^{2}+\left\|A_{h} e^{n}\right\|_{0}^{2}\right)\left\|d_{t} u_{h}^{n}\right\|_{0}^{2} \tau .
\end{aligned}
$$

Combining these inequalities with (6.33) gives

$$
\begin{aligned}
\sigma^{4-\alpha}\left(t_{n}\right)\left\|d_{t} e^{n}\right\|_{0}^{2} & -\sigma^{4-\alpha}\left(t_{n-1}\right)\left\|d_{t} e^{n-1}\right\|_{0}^{2} \leq \sigma^{4-\alpha}\left(t_{n}\right)\left(\frac{2}{\nu}\right)^{3} c_{0}^{4} \gamma_{0}^{2}\left\|u_{h}\left(t_{n}\right)\right\|_{1}^{4}\left\|d_{t} e^{n}\right\|_{0}^{2} \tau \\
& +\left(\sigma^{3-\alpha}\left(t_{n-1}\right)\left\|A_{h} e^{n-1}\right\|_{0}^{2}+\sigma^{3-\alpha}\left(t_{n}\right)\left\|A_{h} e^{n}\right\|_{0}^{2}\right) \sigma\left(t_{n}\right)\left\|d_{t} u_{h}^{n}\right\|_{0}^{2} \tau \\
& +c \sigma^{3-\alpha}\left(t_{n-1}\right)\left\|d_{t} e^{n-1}\right\|_{0}^{2} \tau+c \sigma^{4-\alpha}\left(t_{n}\right)\left\|A_{h}^{-1 / 2} P_{h} d_{t} E_{n}\right\|_{0}^{2} \tau .
\end{aligned}
$$

Summing (6.34) from 3 to $m$ and using Theorem 3.3, Theorem 4.1, Lemma 6.1, Lemma 6.3 with $m=1,2$ and Lemma 6.4 , we obtain

$$
\sigma^{4-\alpha}\left(t_{m}\right)\left\|d_{t} e^{m}\right\|_{0}^{2} \leq \kappa \tau^{2}, 1 \leq m \leq N .
$$


Moreover, we deduce from (6.2), (3.10) and Lemma 3.1 that

$$
\begin{aligned}
\left\|E_{1}\right\|_{0} & \leq\left\|u_{h t}\left(t_{1}\right)\right\|_{0}+\tau^{-1 / 2}\left(\int_{t_{0}}^{t_{1}}\left\|u_{h t}\right\|_{0}^{2} d t\right)^{1 / 2} \\
& +\frac{1}{2}\left(G^{1 / 2}\left(u_{h}^{0}\right)+G^{1 / 2}\left(u_{h}^{1}\right)\right)\left\|d_{t} u_{h}^{1}\right\|_{1} \tau,
\end{aligned}
$$

which yields

Using (5.7), Theorem 3.3 and Theorem 4.2 in (6.36), we obtain

$$
\sigma^{4-\alpha}\left(t_{1}\right)\left\|E_{1}\right\|_{0}^{2} \leq \kappa \tau^{2} .
$$

By (3.4), (3.10), (6.2) and Lemma 3.1, we deduce

$$
\begin{aligned}
\left\|\eta^{m}\right\|_{0} & \leq c\left(\left\|d_{t} e^{m}\right\|_{0}+\left\|e^{m}\right\|_{1}\right)+c\left\|e^{m}\right\|_{1}\left(\left\|u_{h}\left(t_{m}\right)\right\|_{1}+\left\|u_{h}^{m}\right\|_{1}\right) \\
& +c\left\|E_{m}\right\|_{0},
\end{aligned}
$$

which together with Theorems 3.3 and 4.1 yield

$$
\begin{aligned}
\sigma^{4-\alpha}\left(t_{m}\right)\left\|\eta^{m}\right\|_{0}^{2} & \leq \kappa \sigma^{4-\alpha}\left(t_{m}\right)\left\|d_{t} e^{m}\right\|_{0}^{2} \\
& +\kappa \sigma^{3-\alpha}\left(t_{m}\right)\left\|e^{m}\right\|_{1}^{2}+\sigma^{4-\alpha}\left(t_{m}\right)\left\|E_{m}\right\|_{0}^{2}
\end{aligned}
$$

Using (6.35), (6.37), Lemma 6.1 and Lemma 6.4 in (6.38) yields

$$
\sigma^{4-\alpha}\left(t_{m}\right)\left\|\eta^{m}\right\|_{0}^{2} \leq \kappa \tau^{2}, 1 \leq m \leq N
$$

Combining (6.39) with Lemma 6.3 and Lemma 6.4 yields the following error estimates results.

Theorem 6.5. Under the assumptions of Theorem 4.1, the following error estimates hold:

$$
\begin{array}{r}
\sigma^{2-\alpha}\left(t_{m}\right)\left\|u_{h}\left(t_{m}\right)-u_{h}^{m}\right\|_{0}^{2}+\sigma^{3-\alpha}\left(t_{m}\right)\left\|u_{h}\left(t_{m}\right)-u_{h}^{m}\right\|_{1}^{2} \leq \kappa \tau^{2}, t_{m} \in(0, T], \\
\sigma^{4-\alpha}\left(t_{m}\right)\left\|p_{h}\left(t_{m}\right)-p_{h}^{m}\right\|_{0}^{2} \leq \kappa \tau^{2}, t_{m} \in(0, T] .
\end{array}
$$

Remark. Combining Theorem 6.5 with (3.19) yields (1.11)-(1.13).

\section{ACKNOWLEDGEMENTS}

The author would like to thank the referee for valuable comments which helped us to improve the result of this paper.

\section{REFERENCES}

[1] R. A. Adams, Sobolev Spaces, Academic Press, New York, 1975. MR0450957 (56:9247)

[2] A. Ait Ou Ammi And M. Marion, Nonlinear Galerkin methods and mixed finite elements: Two-grid algorithms for the Navier-Stokes equations, Numer. Math., 68 (1994), pp. 189-213. MR.1283337 (95c:65174)

[3] G. A. BAKER, Galerkin approximations for the Navier-Stokes equations, manuscript, Harvard University, Cambridge, MA, 1976.

[4] G. A. Baker, V. A. Dougalis, and O. A. Karakashian, On a high order accurate fully discrete Galerkin approximation to the Navier-Stokes equations, Math. Comp., 39 (1982), pp. 339-375. MR669634 (84h:65096) 
[5] J. Bercovier and O. Pironneau, Error estimates for finite element solution of the Stokes problem in the primitive variables, Numer. Math., 33 (1979), pp. 211-224. MR549450 (81g:65145)

[6] J. R. Cannon and Yanping Lin, A priori $L^{2}$ error estimates for finite-element methods for nonlinear diffusion equations with memory, SIAM J. Numer. Anal., 27 (1990), pp. 595-607. MR1041253 (91b:65118)

[7] P. G. Ciarlet, The Finite Element Method for Elliptic Problems, North-Holland, Amsterdam, 1978. MR0520174 (58:25001)

[8] W. E And J.-G. LiU, Projection methods I: Convergence and numerical boundary layers, SIAM J. Numer. Anal., 32 (1995), 1017-1057. MR1342281(96e:65061)

[9] G. Fairweather, H. Ma And W. Sun, Orthogonal Spline Collocation Methods for the NavierStokes Equations in Stream Function and Vorticity Formulation, submitted.

[10] V. Girault And P. A. Raviart, Finite Element Method for Navier-Stokes Equations: Theory and algorithms, Springer-Verlag, Berlin, Heidelberg, 1987. MR851383 (88b:65129)

[11] Yinnian He, Stability and error analysis for a spectral Galerkin method for the Navier-Stokes equations with $\mathrm{H}^{2}$ or $\mathrm{H}^{1}$ initial data, Numer. Methods for PDEs, 21 (2005), pp. 875-904. MR:2154224 (2006d:65108)

[12] YinNian HE, Stability and error analysis for a spectral Galerkin method for the NavierStokes equations with with $L^{2}$ initial data, Numer. Methods for PDEs, 24 (2008), pp. 79-103. MR2371349

[13] Yinnian He and Kaitai Li, Convergence and stability of finite element nonlinear Galerkin method for the Navier-Stokes equations, Numer. Math., 79 (1998), pp. 77-106. MR1608417 (99c:65165)

[14] Yinnian He and Kaitai Li, Nonlinear Galerkin method and two-step method for the NavierStokes equations, Numer. Methods for PDEs, 12 (1996):283-305. MR:1388441 (97g:65192)

[15] Yinnian HE, Two-level method based on finite element and Crank-Nicolson extrapolation for the time-dependent Navier-Stokes equations, SIAM J. Numer. Anal., 2003, 41(4):1263-1285. MR2034880 (2004k:65173)

[16] Yinnian He AND K. M. Liu, A multi-level finite element method for the time-dependent Navier-Stokes equations, Numer. Methods for PDEs, 21 (2005), pp. 1052-1068. MR 2169167 (2006h:76079)

[17] Yinnian He, K. M. Liu And Weiwei Sun, Multi-level spectral Galerkin method for the Navier-Stokes equations I: spatial discretization, Numer. Math., 101 (2005), pp. 501-522. MR2194826 (2006j:76089)

[18] YinNian He, YanPing Lin And Weiwei Sun, Stabilized finite element methods for the nonstationary Navier-Stokes problem, Discrete and Continuous Dynamical Systems-Series B, 6 (2006), pp. 41-68. MR2172195 (2006g:65152)

[19] Yinnian He And WeIWEI Sun, Stability and convegence of the Crank-Nicolson/AdamsBashforth scheme for the time-dependent Navier-Stokes equations, SIAM J. Numer. Anal., 2007, 45(2):837-869. MR2300299

[20] Yinnian He, Optimal error estimate of the penalty finite element method for the timedependent Navier-Stokes problem, Mathmatics of Computation, 74 (2005), pp. 1201-1216. MR2136999 (2006i:65150)

[21] Yinnian He, Huanling Miao, R. M. M. Mattheij and Zhangxin Chen Numerical analysis of a modified finite element nonlinear Galerkin method, Numer. Math., 97 (2004), pp. 725-756 MR2127930 (2005m:65188)

[22] J. G. HeYwOOd AND R. RANnAcher, Finite-element approximations of the nonstationary Navier-Stokes problem. Part I: Regularity of solutions and second-order spatial discretization, SIAM J. Numer. Anal., 19 (1982), pp. 275-311. MR650052 (83d:65260)

[23] J. G. HeYwood And R. RAnNaCher, Finite-element approximations of the nonstationary Navier-Stokes problem. Part IV: Error estimates for second-order time discretization, SIAM J. Numer. Anal., 27 (1990), pp. 353-384. MR:1043610 (92c:65133)

[24] A. T. Hill And E. SüLI, Approximation of the global attractor for the incompressible NavierStokes equations, IMA J. Numer. Anal., 20 (2000), pp. 633-667. MR.1795301 (2001j:37138)

[25] E. Issacson and H. B. Keller, Analysis of Numerical Methods, Wiley, New York, 1966. MR0201039 (34:924) 
[26] H. Johnston And J.-G. Liu, Accurate, stable and efficient Navier-Stokes slovers based on explicit treatment of the pressure term, J. Computational Physics, 199 (2004), pp. 221-259. MR.2081004 (2005b:76093)

[27] J. Kim AND P. MoIN, Application of a fractional-step method to incompressible Navier-Stokes equations, J. Comput. Phys., 59 (1985), pp. 308-323. MR796611(87a:76046)

[28] R. B. Kellogg and J. E. Osbonn, A regularity result for the Stokes problem in a convex polygon, J. Functional Anal., 21 (1976), pp. 397-431. MR0404849(53:8649)

[29] S. LARsson, The long-time behavior of finite-element approximations of solutions to semilinear parabolic problems, SIAM J. Numer. Anal., 26 (1989), pp. 348-365. MR987394 (90g:65124)

[30] YANPING LiN, Galerkin methods for nonlinear parabolic integrodifferential equations with nonlinear boundary conditions, SIAM J. Numer. Anal., 27 (1990), pp. 608-621. MR1041254 (91e:65128)

[31] Heping Ma and Weiwei Sun, Optimal error estimates of the Legendre Petro-Galerkin and pseudospectral methods for the generalized Korteweg-de Vries Equation, SIAM J. Numer. Anal., 39 (2001), pp. 1380-1394. MR.1870846(2002k:65162)

[32] M. Marion and R. Temam, Navier-Stokes equations: Theory and approximation, in: Handbook of Numerical Analysis, Vol. VI, pp. 503-688, North-Holland, Amsterdam, 1998. MR1665429 (2000a:76002)

[33] R. H. Nochetto AND J.-H. Pyo, A finite element Gauge-Uzawa method Part I: Navier-Stokes equations, SIAM J. Numer. Anal., 43 (2005), pp. 1043-1068. MR2177795 (2006m:65206)

[34] J. SHen, Long time stability and convergence for fully discrete nonlinear Galerkin methods, Appl. Anal., 38 (1990), pp. 201-229. MR1116181 (93a:65130)

[35] J. C. Simo AND F. ARMero, Unconditional stability and long-term behavior of transient algorithms for the incompressible Navier-Stokes and Euler equations, Comput. Methods Appl. Mech. Engrg., 111 (1994), pp. 111-154. MR1259618 (94k:76078)

[36] R. Temam, Navier-Stokes Equations, Theory and Numerical Analysis, 3rd ed., NorthHolland, Amsterdam, 1983. MR769654 (86m:76003)

[37] F. TONE, Error analysis for a second scheme for the Navier-Stokes equations, Applied Numerical Mathematics, 50 (2004), pp. 93-119. MR.2060828 (2005b:65101)

Faculty of Science, Xi'an Jiaotong University, Xi'an 710049, People's Republic of CHINA

E-mail address: heyn@mail.xjtu.edu.cn 